\title{
Molecular Orientation of Conjugated Polymer Chains in Nanostructures and Thin Films: Review of Processes and Application to Optoelectronics
}

\author{
Varun Vohra and Takuya Anzai \\ Department of Engineering Science, University of Electro-Communications, Tokyo, Japan \\ Correspondence should be addressed to Varun Vohra; varun.vohra@uec.ac.jp
}

Received 29 August 2016; Accepted 12 February 2017; Published 21 March 2017

Academic Editor: Christian Brosseau

Copyright ( 2017 Varun Vohra and Takuya Anzai. This is an open access article distributed under the Creative Commons Attribution License, which permits unrestricted use, distribution, and reproduction in any medium, provided the original work is properly cited.

\begin{abstract}
Semiconducting polymers are composed of elongated conjugated polymer backbones and side chains with high solubility and mechanical properties. The combination of these two features results in a high processability and a potential to orient the conjugated backbones in thin films and nanofibers. The thin films and nanofibers are usually composed of highly crystalline (high charge transport) and amorphous parts. Orientation of conjugated polymer can result in enhanced charge transport or optical properties as it induces increased crystallinity or preferential orientation of the crystallites. After summarizing the potential strategies to exploit molecular order in conjugated polymer based optoelectronic devices, we will review some of the fabrication processes to induce molecular orientation. In particular, we will review the cases involving molecular and interfacial interactions, unidirectional deposition processes, electrospinning, and postdeposition mechanical treatments. The studies presented here clearly demonstrate that process-controlled molecular orientation of the conjugated polymer chains can result in high device performances (mobilities over $40 \mathrm{~cm}^{2} \cdot \mathrm{V}^{-1} \cdot \mathrm{s}^{-1}$ and solar cells with efficiencies over 10\%). Furthermore, the peculiar interactions between molecularly oriented polymers and polarized light have the potential not only to generate low-cost and low energy consumption polarized light sources but also to fabricate innovative devices such as solar cell integrated LCDs or bipolarized LEDs.
\end{abstract}

\section{Introduction}

Conjugated polymers have been raising an increasing interest over the past two decades as they represent a low-cost alternative to silicon technology and can therefore be considered as the most promising candidates for the next generation of optoelectronic devices [1, 2]. In fact, the objective of conjugated polymer based electronics is to provide similar performances to silicon-based electronics using solvent and roll-to-roll compatible processes [3]. However, depending on which process conditions and deposition process are employed, the nanoscale morphology of the thin conjugated polymer films can vary enormously [4]. A large number of scientific papers have been published on the effect of the process conditions over the resulting morphology, trying to understand the correlation between morphology and performances [5-7]. In fact, understanding these correlations from both theoretical and experimental points of view represents a major achievement to increase the performances of conjugated polymer devices such as polymer solar cells (PSCs), polymer light-emitting devices (PLEDs), or polymer field-effect transistors (PFETs).

Soluble conjugated polymers are usually composed of a rigid conjugated backbone and flexible side chains which provide the potential to generate highly crystalline structures (mostly through $\pi-\pi$ interactions) while maintaining high solubility in organic solvents, flexibility, processability, and films formation properties. To fabricate thin films with these complimentary properties, a balance between deformability and charge transport needs to be obtained [8]. Another peculiar property of conjugated polymers is that, being macromolecules, the long chains can be easily oriented which may lead to enhanced polymer crystallinities or enhanced directional charge mobility. Charge transport in conjugated 


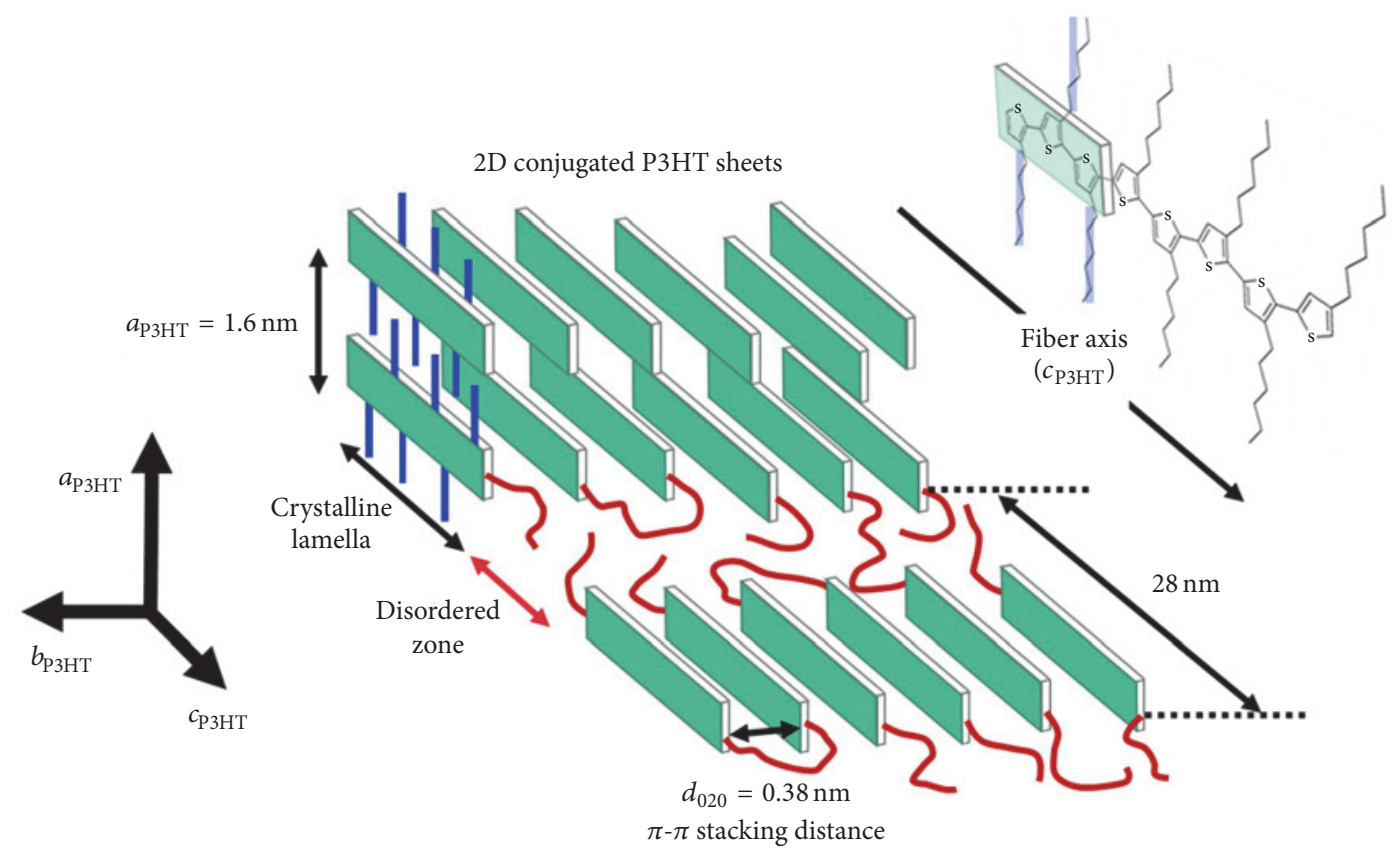

(a)

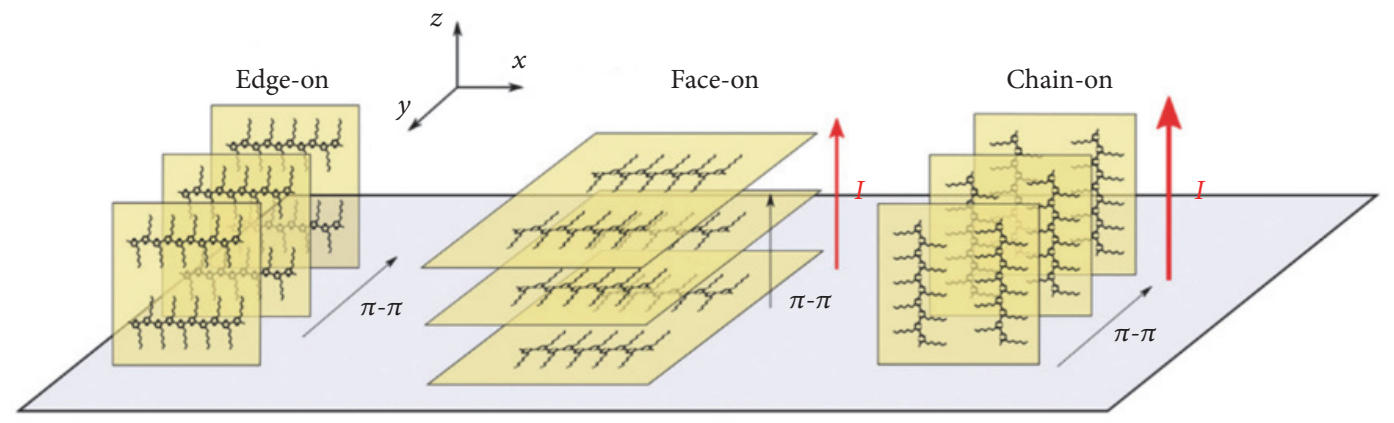

(b)

FIGURE 1: Schematic representation of molecular order (a) and crystallite orientation (b) in P3HT conjugated polymer thin films. This figure was adapted with permission from [59, 70]. Copyright 2009, Lactualité chimique. Copyright 2016, John Wiley and Sons.

polymers is a complicated phenomenon involving multiple complimentary mechanisms. Conjugated polymers used in optically and electronically active thin films generally display semicrystalline properties and the resulting films thus contain ordered and disordered zones (Figure 1) [4, 8-12].

The main mechanisms for conjugated polymer solid state charge transport can be separated into two groups, namely, intrachain and interchain transport mechanisms. As intrachain transport occurs through covalently bond conjugated units, the charge transport is highest along the conjugated backbone. While the intrachain mobility represents the highest mobility direction (over short distances and limited by lamella dimensions), interchain charge hopping through $\pi-\pi$ interactions in the crystalline portions of the thin films also appears to be a major contribution to charge transport in conjugated polymers devices (Figure 1) [10]. The resulting anisotropy in charge transport is further emphasized when taking into account the relative orientation of the polymer chains with respect to the substrate [6]. The different crystallite orientations are referred to as edge-on, face-on, and chain-on, which correspond to the planar conjugated backbone lying perpendicular, parallel, and standing perpendicular to the substrate, respectively (Figure 1). Note that the molecular representations in Figure 1 were prepared using poly(3-hexylthiophene) (P3HT), the most widely studied semiconducting polymer, but similar models can be obtained for every planar conjugated backbone polymer.

Furthermore, upon conformational elongation of the conjugated polymer chains along a single direction, one can also align their transition dipole moments which results in polarized absorption or emission properties. Polarization is an aspect of light that is often overlooked when designing optical systems and devices. Polarized light is widely used for applications such as lenses for sunglasses (UV-polarized) or fabrication of next-generation 3D displays or liquid crystal 


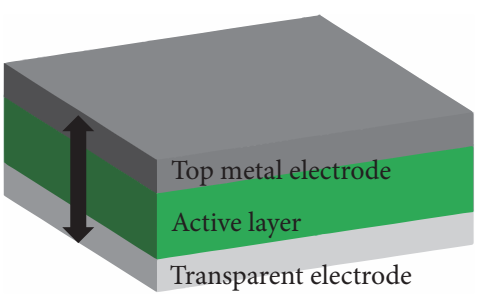

Vertical device architecture

(PLEDs, PSCs)

Charge transport direction

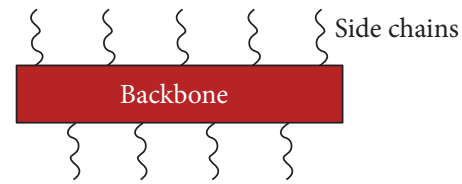

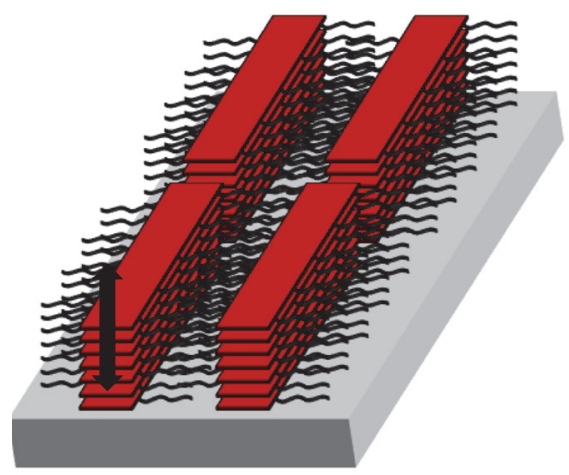

Face-on crystallites

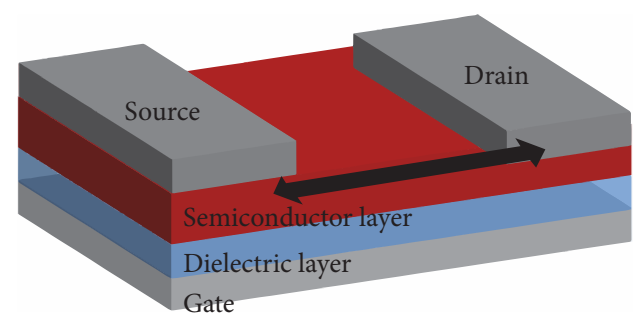

Horizontal device architecture

(PFETs) (a)

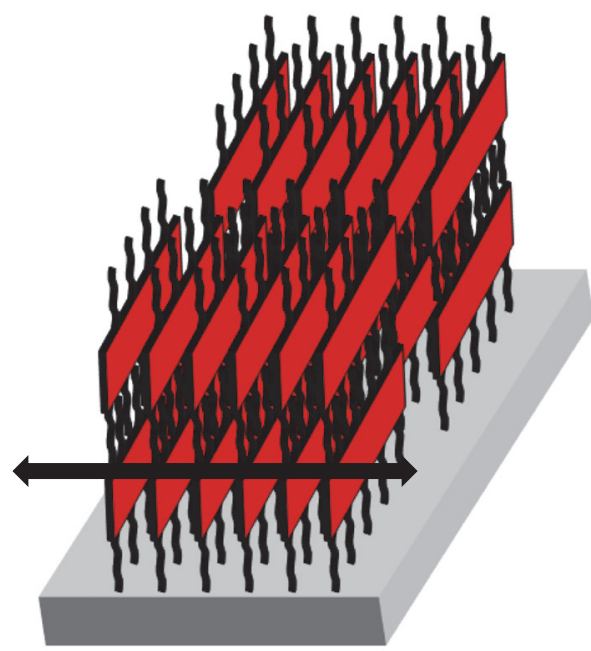

Edge-on crystallites

(b)

FIGURE 2: Schematic representation of (a) the typical device architectures for conjugated polymer electronics and (b) preferential charge transport direction in face-on and edge-on crystallites.

displays but also as polarized light sources in analytical scientific equipment. Therefore, a particular effort has been given over the past few years to fabricate low power consumption highly polarized organic or inorganic light sources [13-16]. In polymer conjugated systems, which can be easily oriented, polarized electroluminescence has been demonstrated for around two decades [17]. Furthermore, integrating polarized components into optoelectronic devices may lead to the development of innovative technologies and concepts [18].

In conjugated polymer thin films and devices, enhanced directional mobility and polarization can be achieved using a variety of processes such as epitaxial growth [19], LangmuirBlodgett deposition [20], unidirectional deposition [21], mechanical alignment [17, 18], electrospinning [22], or nanoconfinement [23]. In this paper, after summarizing the potential strategies to improve device performances through molecular orientation, we will review some of the methods to induce molecular orientation of conjugated polymer chains at the nanoscale and correlate the resulting morphologies with the optical and electrical properties of the films and devices.

\section{Improving the Device Performances through Molecular Orientation}

2.1. Device Architecture, Molecular Orientation, and Charge Transport. Conjugated polymer devices can generally be divided into two types of architecture: horizontal and vertical architectures (Figure 2). Although recent introduction of new light-emitting device architectures (organic light-emitting field-effect transistors and organic light-emitting electrochemical cells) demonstrates that horizontal architecture light-emitting devices can be fabricated, PLEDs, in which the charge transport relies solely on the intrinsic properties of the polymer material, are commonly fabricated in a vertical device architecture (emitting layer sandwiched between two electrodes with, potentially, insertion of hole- and electrononly layers). Similarly, PSCs, in which the charges should quickly move to the respective electrodes, are limited to vertical device architectures. In fact, as these two types of device rely on the intrinsic transport properties of the polymer, the distance over which the charges travel should be 


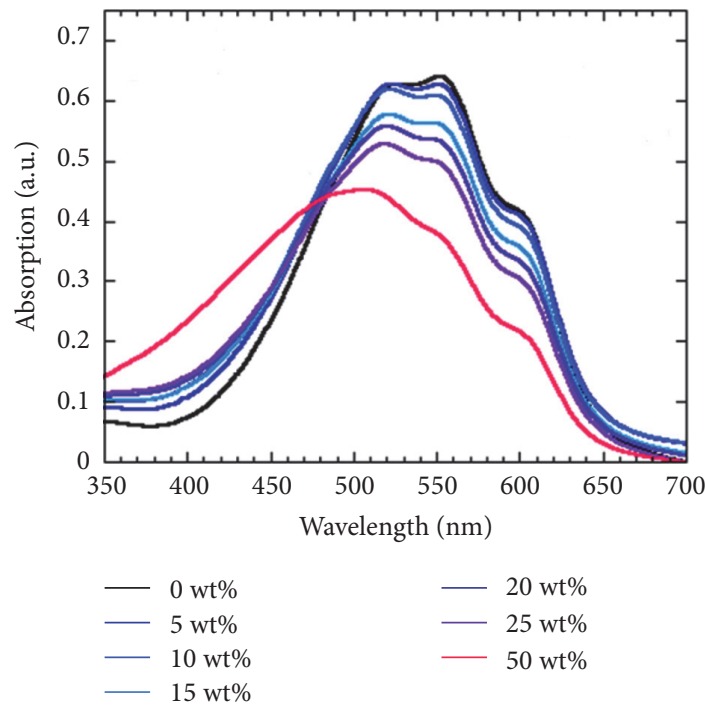

(a)

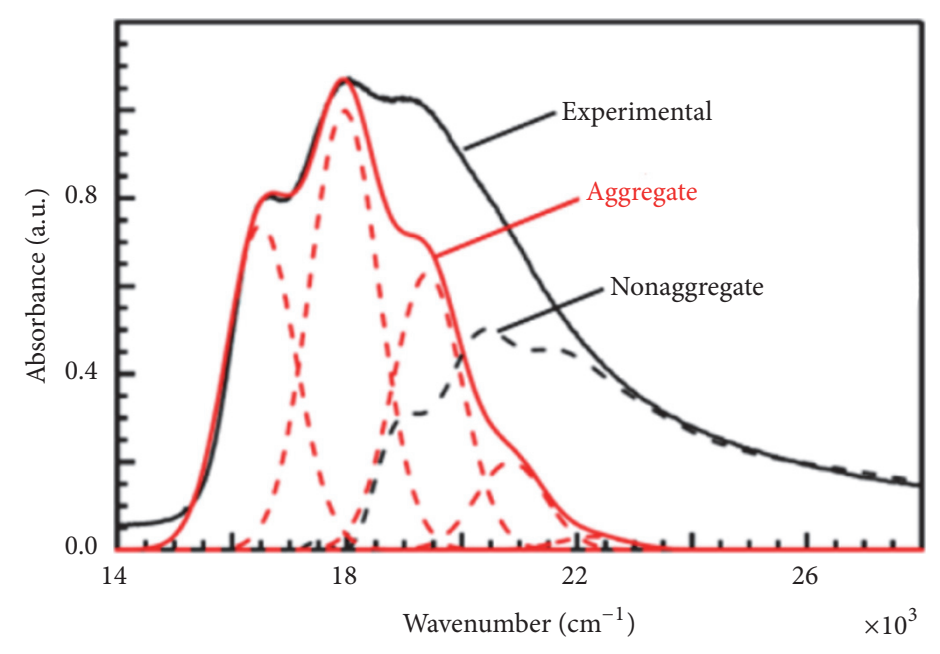

(b)

FIGURE 3: Absorption spectra of P3HT (a) with various concentrations of added regiorandom P3HT and (b) decomposed with contributions from the aggregate and nonaggregate phases in the thin films. This figure was adapted with permission from [32, 33]. Copyright 2012, AIP Publishing LLC. Copyright 2015, American Chemical Society.

limited to a few hundreds of nanometers at the maximum. On the other hand, in the on state of PFETs, charges need to move from the source to the drain and high horizontal transport properties between these two electrodes are necessary. As mentioned above, the charge transport over distances that exceed a few tens of nanometers is essentially governed by charge hopping in semicrystalline conjugated polymers.

As charge hopping occurs through $\pi-\pi$ interactions, faceon and edge-on crystallite orientations will favor vertical and horizontal charge transports, respectively. Note that, as chainon consists of a particularly rare case, we did not represent it in Figure 2. However, the charge transport properties of chain-on crystallites should enhance horizontal charge transport, similarly to edge-on crystallites and potentially vertical charge transport over short distances. The effect of molecular orientation has been extensively studied for small organic molecules and/or single organic crystal field-effect transistors with large mobility increases observed for oriented thin films compared to amorphous or randomly oriented ones [24]. Similarly, the effect of molecular orientation is a well-known phenomenon in both vertical architecture PLEDs and PSCs. In fact, in PSCs, the fill factor (FF) of the devices, which represents the capacity of the photogenerated charges to percolate to their respective electrodes, can show extremely high values (over $70 \%$ ) by inducing high concentrations of face-on crystallites $[25,26]$. Similarly, in small molecules and PLEDs, the external quantum efficiencies (EQE) show a large enhancement upon orientation of either the charge injection or the emitting layer $[27,28]$. Surprisingly, in single crystal light-emitting devices, rather low EQEs were obtained (lower than $0.0003 \%$ ) even though single crystals provide a higher degree of order compared to the crystalline phases in conjugated polymer thin films [29]. This may be due to the inadequate stacking orientation in the single crystals for vertical device architectures. Nevertheless, some single crystal devices display very peculiar optical properties (bipolarity) [16] which also represents one of the major advantages of directional organization of organic molecules (including conjugated polymers) as we will discuss in the following section.

\subsection{Optical Effects in Molecularly Oriented Solid State Con-} jugated Molecules. Light-matter interactions has been extensively studied over decades and it is now commonly accepted that organic molecules (including conjugated polymers) display high optical absorption properties which can be easily tuned using either molecular design or nanoassembly $[30,31]$. For instance, in the case of P3HT, major differences can be found in the absorption spectra of molecularly aggregated regioregular $\mathrm{P} 3 \mathrm{HT}$ and amorphous zones or regiorandom P3HT, not only in terms of absorption intensity but also in spectral shape (Figure 3) [32, 33].

In PSCs, a strong absorption is necessary to generate more excitons and, consequently, a larger amount of photogenerated charges. Furthermore, if unidirectional molecular organization can be achieved in the active polymer layer of semitransparent PSCs, only a fraction of light (polarized parallel to the polymer transition dipole moment) will be absorbed and the remaining transmitted light can be used for other purposes (e.g., absorption by a second perpendicularly organized active layer, in LCDs [18] or simply in semitransparent photovoltaic windows).

Polarization of light is an important aspect of new and future display technologies. In fact, not only absorption but 


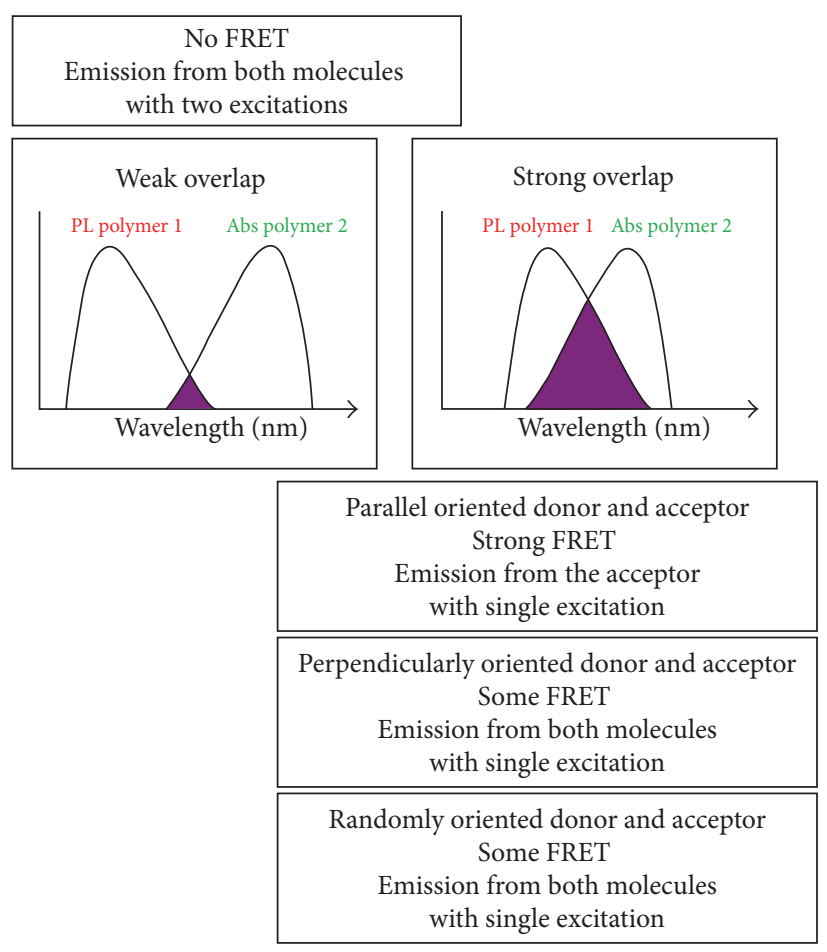

(a)
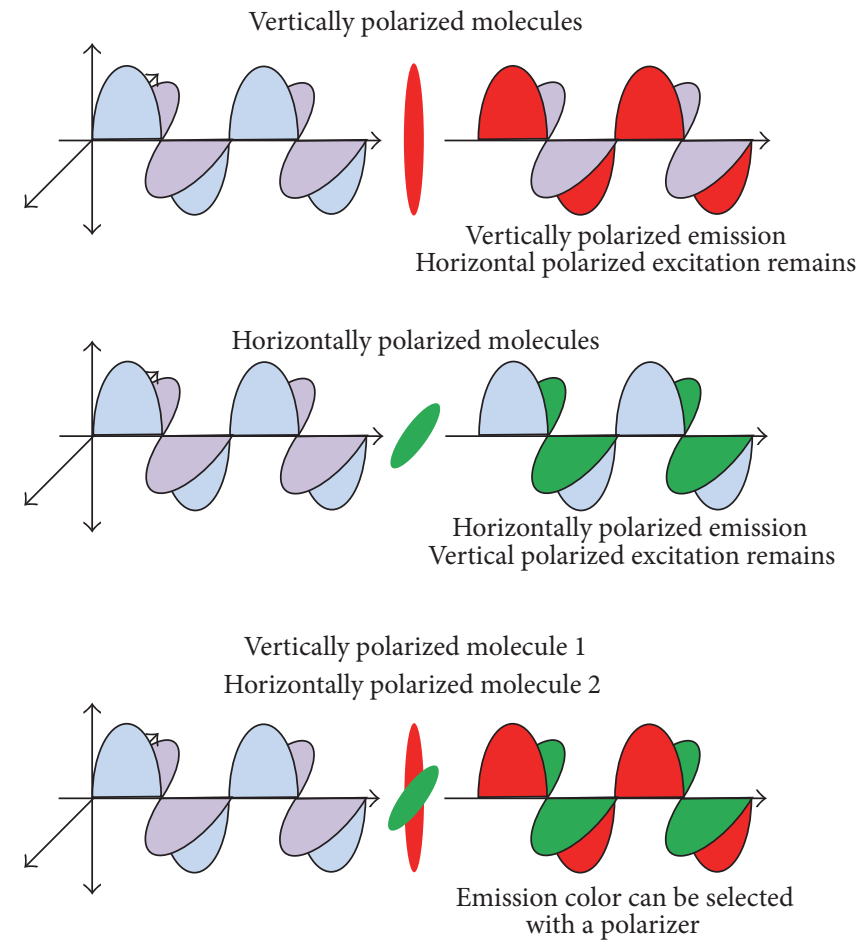

(b)

FIGURE 4: Strategies to control the emission properties of thin films containing (a) donor and acceptor molecules for FRET or (b) molecules having their transition dipole moment oriented along the $y$-and $z$-axis.

also generation of polarized light and interactions between light-absorbing and light-emitting unidirectionally oriented molecules provides key aspects to design technologies such as tunable color thin films, high efficiency optical communications systems, and/or high efficiency liquid crystal displays (LCDs) based on polarized PLEDs backlight. One of the strategies to design color tunable systems is based on the use of controlled Förster resonant energy transfer (FRET) between fluorescent donor and acceptor molecules. The amount of energy transferred strongly depends on the overlap integral between the emission spectrum of the donor and the absorption spectrum of the acceptor. However, the alignment of the transition dipole moments of the two molecules also strongly influences the transfer ratio which is maximized for parallel donor and acceptor transition dipole moments [34]. Therefore, as summarized in Figure 4, tuning the emission properties and color of PLEDs could be easily achieved through specific relative orientations active layers or thin films containing more than one active molecule. Furthermore, the generation of PLEDs exhibiting two perpendicular preferential molecular orientations also represents a simple but efficient strategy to fabricate bicolor emitting devices in which the emission color can be easily selected through the rotation of a polarizer $[16,17]$. Additionally, orientation of the molecules with respect to the substrate in light-emitting devices may lead to enhanced light-outcoupling properties which, in turn, could generate higher performances devices [35].

\section{Processes to Induce Molecular Orientation and Their Effect on Optoelectronic Device Properties}

3.1. Molecular Orientation Induced by Materials Interaction during Spin-Coating. The most common process used to deposit active layers and thin films for polymer electronics is spin-coating. Spin-coating generates homogeneous films using centrifugal forces and the film thicknesses can be easily adjusted by changing either the solution concentration or the rotation speed. However, to obtain homogeneous films, interactions between the substrate and the solution play an essential role. These interactions can also be used to generate preferential orientation of the polymer chains as they interact with the substrate during the deposition process. For instance, a large number of studies demonstrated that using self-assembled monolayers to modify the substrate surfaces, a large increase in performances of PFETs can be observed. Kim et al. used the self-assembled monolayer approach to functionalize the substrate surface with either $-\mathrm{NH}_{2},-\mathrm{OH}$, or $-\mathrm{CH}_{3}$ groups and observed their effect on the molecular orientation of P3HT and the resulting field-effect mobility [36]. The results clearly demonstrate that while interactions with $-\mathrm{CH}_{3}$ functionalized surfaces favor face-on crystallite orientation, $-\mathrm{NH}_{2}$ functionalized surfaces, on the other hand, generate a larger concentration of edge-on crystallites. Consequently, the field-effect mobility increased 


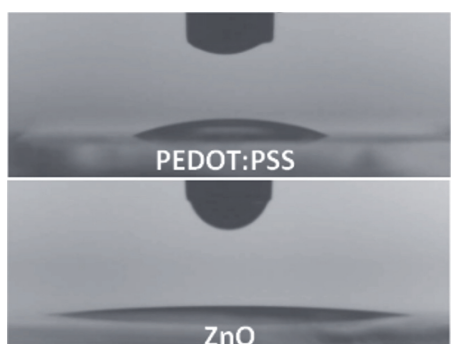

(a)

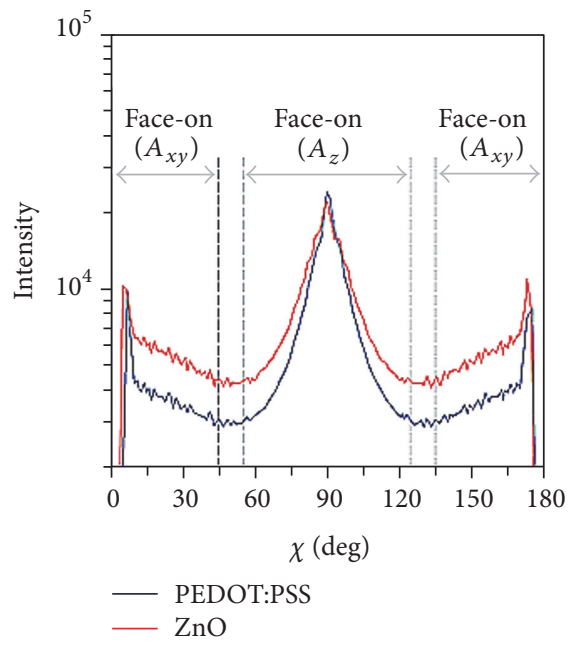

(b)

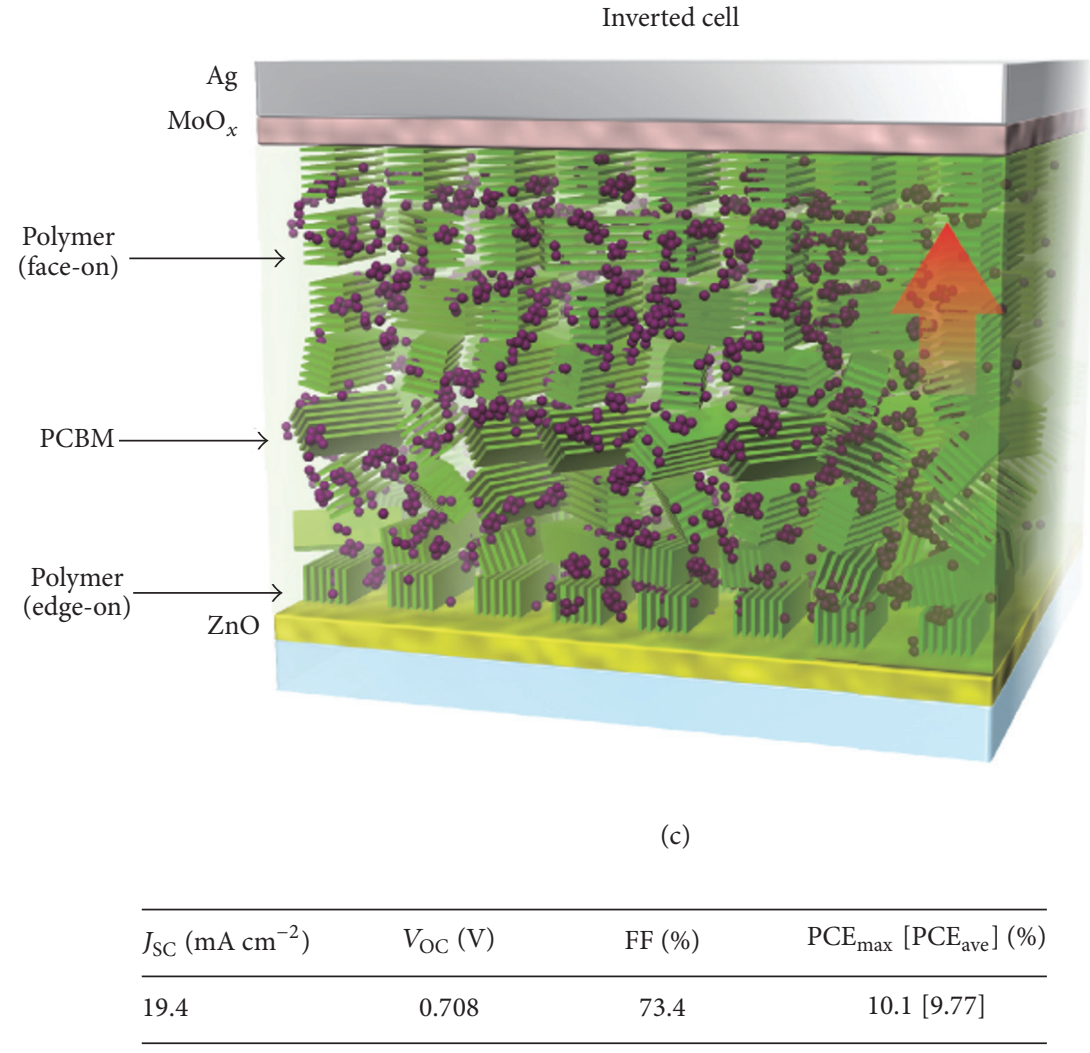

(d)

FIGURE 5: (a) Wettability of dichlorobenzene on PEDOT:PSS and ZnO surfaces; (b) variation in face-on/edge-on crystallite ratio in PNTz4T:PC ${ }_{71}$ BM films deposited from dichlorobenzene on PEDOT:PSS and ZnO surfaces; (c) schematic representation and (d) photovoltaic parameters of inverted PSCs with PNTz4T:PC ${ }_{71}$ BM active layers. This figure was adapted with permission from [25]. Copyright 2015, Nature Publishing Group.

approximately 3.5 times when switching from $-\mathrm{CH}_{3}$ to $-\mathrm{NH}_{2}$ functionalized surfaces, with values of 0.08 and $0.28 \mathrm{~cm}^{2} \cdot \mathrm{V}^{-1} \cdot \mathrm{s}^{-1}$, respectively.

While PFETs benefit from a large edge-on crystallite concentration, the fabrication of PSCs with high FFs requires a higher face-on crystallite concentration. PSCs can generally be divided in two types of device architectures, namely, regular and inverted device architectures. Regular and inverted devices are, respectively, prepared using a hole collecting conjugated polymer (e.g., PEDOT:PSS) and electron collecting metal oxide (e.g., $\mathrm{ZnO}$ ) layers coated on the transparent ITO substrates. As the two materials have different surface tensions and surface functions, similarly to the work on PFETs [36], different crystallite orientations should be expected for active layers in regular and inverted device architectures. In fact, in PSCs prepared with Naphtho[1,2-c:5,6-c'] bis[1,2,5]thiadiazole based p-type polymer (PNTz4T):[6,6]-phenyl- $\mathrm{C}_{71}$-butyric acid methyl ester $\left(\mathrm{PC}_{71} \mathrm{BM}\right)$ blend solutions, the active layer solution has different wetting properties on PEDOT:PSS and $\mathrm{ZnO}$ [25]. Consequently, a different amount of face-on and edge-on crystallites is generated in the thin blend films deposited on the ITO substrates with the two different coatings. While active layers coated on PEDOT:PSS provide an overall lower face-on concentration with respect to those coated on $\mathrm{ZnO}$, through a systematic analysis of active layers with increasing thicknesses using 2D-GIXRD, the formation of an edgeon/face-on crystallite vertical concentration gradient for both PEDOT:PSS and $\mathrm{ZnO}$ coated substrates was revealed. As represented on Figure 5, a higher concentration of edgeon crystallites can be found towards the bottom of the film, while face-on crystallites concentrate in the bulk and at the top of the film. As PSCs require increased vertical charge transport from the bulk to the anode, in regular device architectures, the edge-on crystallites at the bottom of the film act as a hole blocking layer lowering the device performances. On the other hand, in inverted devices, this hole blocking layer has a positive effect as it reduces leak current (holes in contact with the cathode) while the faceon crystallites in the bulk and at the top of the film provide fast and efficient hole transport to the anode. The increased overall face-on crystallites concentration and the adequate crystallite orientation gradient in inverted PSCs result in FFs over $70 \%$ and, consequently, the fabrication of PSCs with PCE exceeding the milestone value of $10 \%$.

Furthermore, pure PNTz4T and PNTz4T:fullerene blend films exhibited different crystallite orientations. While the 

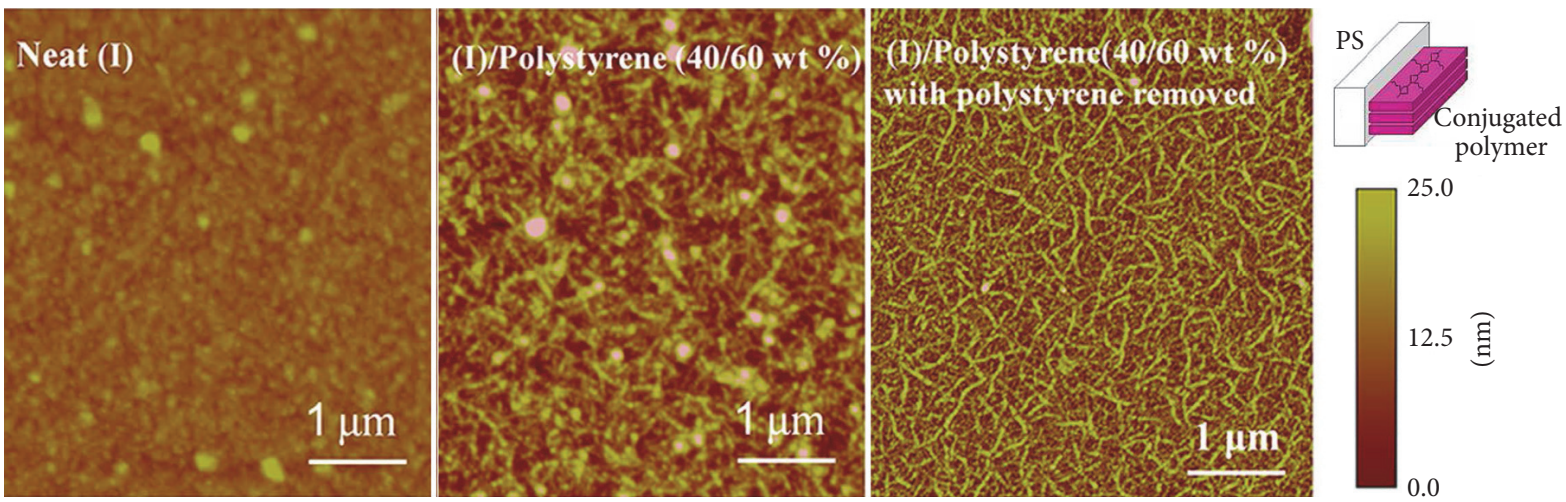

FIGURE 6: Surface morphologies of PDPP-DTT films obtained with and without PS. The inset of the figure represents the molecular order generated at the interface between PS and P3HT. This figure was adapted from [37, 38]. Copyright 2016, Nature Publishing Group. Copyright 2012, Royal Society of Chemistry.

pure film showed a large concentration of edge-on crystallites, the blends displayed a larger amount of face-on crystallites [26]. As pure PNTz4T and blend films are used for PFETs and PSCs, respectively, the crystallite reorientation upon blending proved favorable for PSCs fabrication while a high field-effect mobility of $0.56 \mathrm{~cm}^{2} \cdot \mathrm{V}^{-1} \cdot \mathrm{s}^{-1}$ could be observed in PFETs. These results suggest that interactions between various molecules in the spin-coated solution can strongly influence both the crystallinity and the crystallite orientation in conjugated polymer based thin films. In fact, spin-coating blends of P3HT and polystyrene (PS) also exhibit a different crystallite orientation of $\mathrm{P} 3 \mathrm{HT}$ as compared to pure $\mathrm{P} 3 \mathrm{HT}$ films. Using the interaction between P3HT and PS, the amount of face-on crystallites present on the surface of P3HT thin films can be controlled as P3HT crystallites arrange in a preferential direction at the interface with PS (inset of Figure 6) [37]. A similar approach based on P3HT and PS interactions in aging solution was introduced in 2009 for PFET fabrication and was recently adapted by Lei et al. to enhance the crystallinity and, consequently, the mobility, of a low Mw donor-acceptor polymer (PDPP-DTT) [38, 39]. Unlike the neat PDPP-DTT film, the PS/PDPP-DTT film containing 60 PS wt\% displays a microfiber-like structure which is further emphasized after PS removal (Figure 6) [38]. The PFETs prepared from the blend solutions demonstrate field-effect mobility values up to $8.25 \mathrm{~cm}^{2} \cdot \mathrm{V}^{-1} \cdot \mathrm{s}^{-1}$, which is approximately 6 times higher than the neat film. Furthermore, the threshold voltage of the PFET is reduced from $9.7 \mathrm{~V}$ (neat film) to $1.7 \mathrm{~V}$ (microfibers). These results confirm that a high crystallinity and fiber-like structure can be extremely beneficial for the fabrication of high performance PFETs. Furthermore, as PS is a widely available commercial polymer, this approach could be easily applied to other systems without having to synthesize new molecules and/or perform surface modification chemistry.

However, the approach based on blending with PS as a crystallization medium has one major disadvantage: an additional PS removal step is necessary prior to device finalization. In fact, if crystallite orientation is not taken into account and the objective is solely to increase the polymer crystallinity, this can be easily achieved by using solvents with higher boiling temperatures. Previous results using chlorinated solvents with 4 different boiling temperatures demonstrated that higher performance PSCs can be obtained by using the solid solvent 1,3,5-trichlorobenzene (TCB) as additive for the active layer deposition [40]. Compared to the reference device, the PCE increased more than $30 \%$ upon addition of $10 \mathrm{wt} \%$ of TCB. In fact, this strategy, often referred to as epitaxial growth, has been widely used since 2006 to generate highly crystalline spherulites of conjugated polymers $[19,41]$. The advantage of using TCB is that, once deposited, the solid solvent, which acts as crystallization agent during the deposition process, is naturally sublimated and, unlike PS, no further step of crystallization agent removal is necessary. The other major advantage of this fabrication process is that it can be applied to virtually any polymer as it does not rely on interactions between TCB and the polymer but rather on the formation of TCB fibers on which the conjugated polymer chains are deposited in a compact arrangement. In fact, as represented in Figure 7, this process can be applied to recently developed conjugated polymers such as PDTDPPQT which should exhibit much higher field-effect mobilities compared to P3HT [42]. In their study on PDTDPPQT, Kim et al. fabricated highly crystalline conjugated polymer spherulites which acted as semiconducting layer in PFETs with source and drain electrodes deposited in various position with respect to the center of the spin-coated substrate. In particular, they compared the field-effect mobilities along and across the formed conjugated polymer fibers (Figure 7). High hole mobility values could be observed for transistors placed both along and perpendicular to the conjugated polymer fibers with values reaching 12.04 and $3.66 \mathrm{~cm}^{2} \cdot \mathrm{V}^{-1} \cdot \mathrm{s}^{-1}$ for parallel and perpendicular PFETs, respectively. Note that even though both values are relatively large for conjugated polymer materials, the mobility along the fiber direction displays a 3.5-fold higher value compared to the mobility perpendicular to the fibers. According to their analysis, the direction along the fiber axis corresponds to $\pi$ $\pi$ stacking direction while the direction perpendicular should 


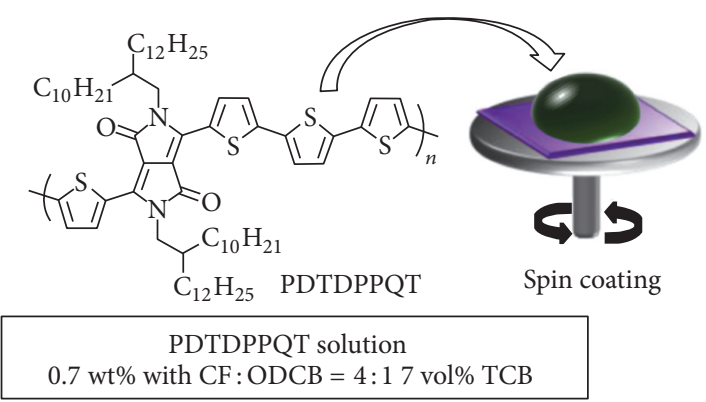

(a)

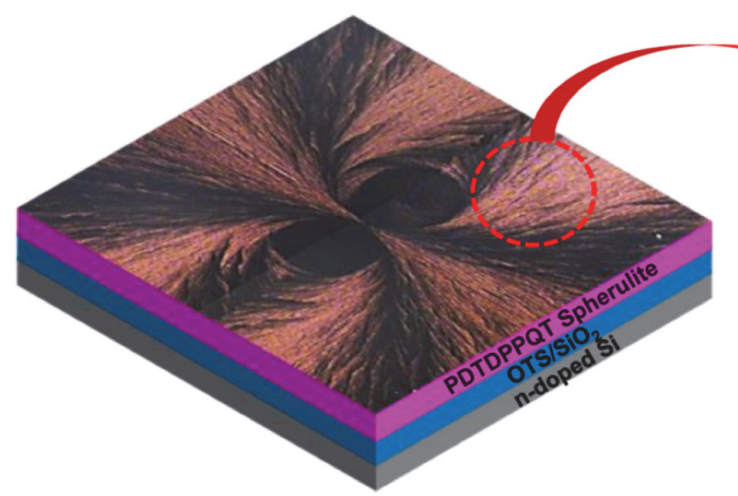

(c)

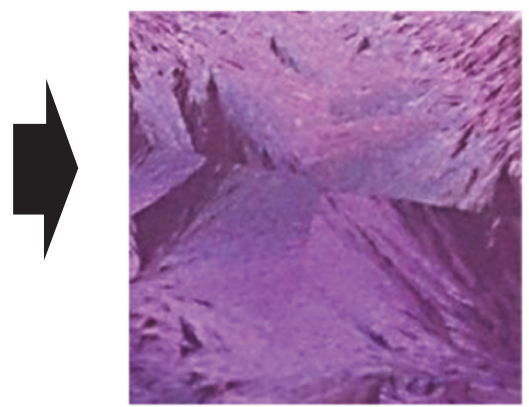

(b)

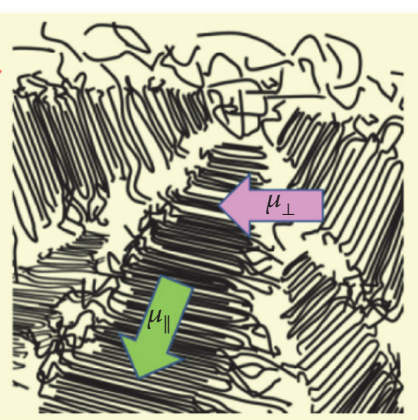

(d)

FIGURE 7: (a) Schematic representation of the spin-coating process with TCB and (b) optical image of the surface of the spin-coated film. Schematic representations of (c) the multilayer device architecture prior to electrode deposition and (d) the molecular order in the spherulites. This figure was reproduced from [42]. Copyrights 2015, American Chemical Society.

be governed mainly by intrachain transport which may occur along more than one polymer chain.

The studies presented in this section all lead to either increased crystallinity of the conjugated polymer thin films or preferential crystallite orientation in the films resulting in higher performing devices. However, as all these results were obtained with spin-coating (radial expansion of the polymer solution), unidirectional orientation of the polymer chain could not be achieved. To achieve some of the strategies presented in Part I, in particular, those related to polarized optical properties, unidirectional processes which induce long range orientation of the polymer chains with respect to the substrate may be necessary.

3.2. Molecular Orientation Induced by Unidirectional Processes. Generation of polarized PLEDs using processes such as the Langmuir-Blodgett technique was first investigated during the second half of the 1990s [43, 44]. This research demonstrated that directional deposition processes have the potential to produce films with polarization ratios (defined as $\left[P_{\|}-P_{\perp}\right] /\left[P_{\|}+P_{\perp}\right]$ where $P_{\|}$and $P_{\perp}$ correspond to photoluminescence intensities parallel and perpendicular to the oriented molecules, resp.) reaching up to 3.5 in poly(pphenylene)-based thin films and between 1.2 and 5.3 in P3HT thin films. Although the device performances for the PLEDs prepared using this technique did not exhibit high performances, these studies introduced the concept of PLED polarization through unidirectional molecular orientation. Moreover, Natali et al. applied Langmuir-Blodgett deposition to PFETs which displayed a mobility anisotropy ratio around 10 as the rigid backbones of P3HT aligned perpendicular to the compression direction [20]. These pioneering works opened the path to a new approach to develop either original PLEDs or high efficiency PFETs and PSCs. In the case of Langmuir-Blodgett films, even though high anisotropy ratios could be observed, this approach is limited to polymers possessing the ideal (amphiphilic) chemical nature and the quality of the films obtained remained rather low, leading to lower device performances as compared to spin-coated films.

In fact, spin-coating corresponds to the most studied deposition process for solution-based polymer electronics up to now. In recent years, the spin-coating deposition process has been modified to generate directional order by developing a new process referred to as off-center spincoating (Figure 8) [21, 45, 46]. During the "classic" spincoating process, the solution deposited or dropped on the surface spreads from the center of the substrate over the entire surface through centrifugal forces generating a homogeneous film as solvent evaporates. As its name indicates, in the off-center spin-coating process, the substrate is placed away from the rotation center. The solution, which still undergoes centrifugal forces, is now spread from the side closer to the center to the other side of the substrate in a quasiunidirectional manner which results in orientation of the 


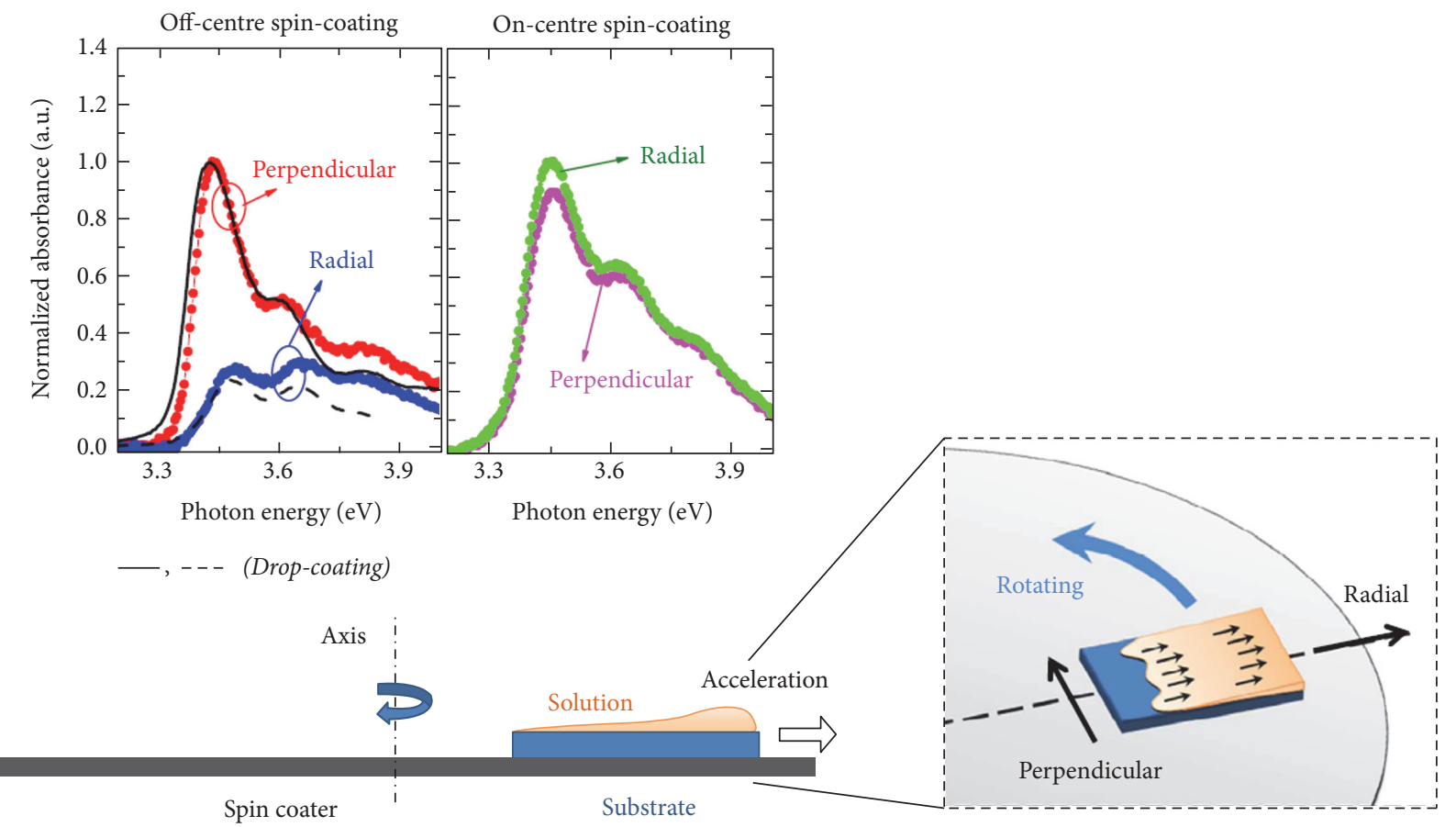

FIGURE 8: Schematic representation of the off-center spin-coating process and comparison between polarized absorption obtained for offcenter and in-center spin-coated films. This figure was adapted with permission from [45]. Copyright 2014, Nature Publishing Group.

conjugated polymer molecules (Figure 8). Preaggregation in solution combined with off-center spin-coating deposition resulted in a conjugated polymer mobility in PFET up to $7.25 \mathrm{~cm}^{2} \cdot \mathrm{V}^{-1} \cdot \mathrm{s}^{-1}$ and a mobility anisotropy ratio of 37 [21]. Furthermore, extremely high mobilities up to $43 \mathrm{~cm}^{2} \cdot \mathrm{V}^{-1} \cdot \mathrm{s}^{-1}$ were obtained by depositing molecules with off-center spincoating which can also exhibit polarized absorption properties or increase the FF of PSCs leading to PCE over $10 \%$ $[45,46]$.

However, even if directional deposition can be achieved, this process is still based on spin-coating which cannot be considered as a roll-to-roll process. Scientists working in the field of organic electronics often emphasize the fact that polymer and organic electronics represent a low-cost alternative to silicon-based electronics. To make this a reality, high productivity roll-to-roll compatible processes are required. The most common solution processes used for active layer deposition in organic electronics are summarized in Figure 9. The top part of Figure 9 represents the substrate-by-substrate processes (spin-coating and Langmuir-Blodgett) while, in the bottom part, typical roll-to-roll compatible processes such as blade-coating and dye-coating are represented. Not only do these roll-to-roll compatible processes allow fully exploiting the potential of conjugated polymers to produce extremely low-cost electronics, but also the deposition is also performed unidirectionally and involves shear forces which may lead to peculiar orientation of the polymer fiber in the deposited thin films. In 2016, Shaw et al. demonstrated the correlation between deposition speed and molecular orientation in thin films deposited using a process they refer to as solution shearing [47]. The process is very similar to blade-coating with the blade tilted at a very low angle $\left(8^{\circ}\right)$ with respect to the substrate. They demonstrate that, at shearing speeds around $0.2 \sim 0.3 \mathrm{~mm} \cdot \mathrm{s}^{-1}$, dichroic ratios up to 7 could be observed with the polymer backbones aligning parallel to the shearing direction. However, in PFETs prepared using solution shearing, no major mobility anisotropy could be observed when comparing PFETs prepared along and perpendicular to the deposition direction.

Similar results were also obtained on conjugated polymer thin films deposited by blade-coating on nanogrooved substrates [48]. These results suggest that a higher degree of crystallinity induced during unidirectional deposition will improve the formation of optically polarized thin films. As we described in the previous part of this review, addition of TCB to the active layer solution for both PFETs and PSCs can be beneficial as it induces a high degree of polymer crystallization. In fact, by combining the unidirectional deposition (dye-coating) and the crystallization agent (TCB) methods, Dörling et al. demonstrated that deposition speed and TCB concentration can control the morphology of the deposited films [49]. At low deposition speeds and high relative TCB concentrations, highly crystalline unidirectional fibrous structures are formed which exhibit optical anisotropy (Figure 10). Using these fibers as base for the production of sequentially deposited PSCs, they were able to fabricate devices displaying a much higher PCE when excited with light polarized along the fiber axis than in the direction perpendicular to the fiber axis.

The formation of nanofibers seems to be beneficial for either the molecular orientation along the fiber and/or to 


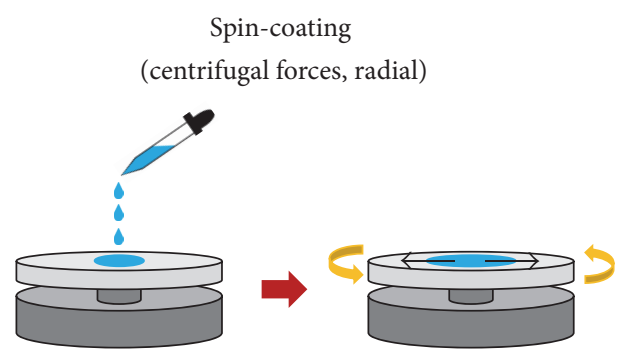

(a)

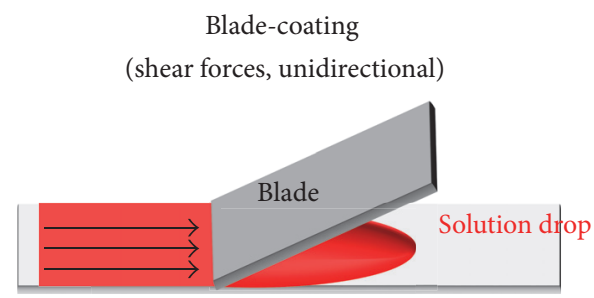

(b)

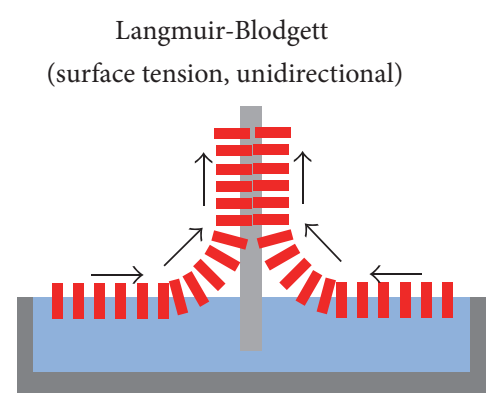

Dye-coating

(shear forces, unidirectional)

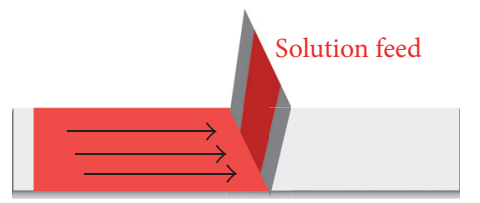

FIGURE 9: Schematic representation of typical substrate-by-substrate (a) and continuous roll-to-roll compatible (b) solution deposition processes.

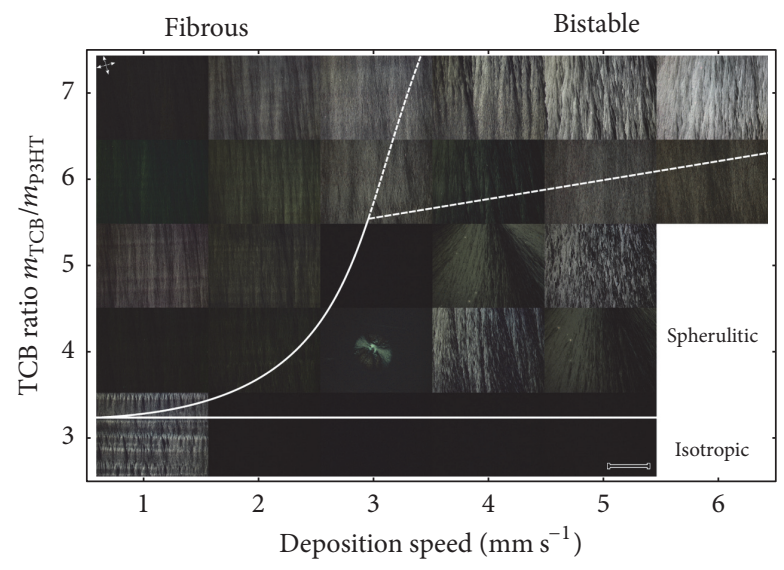

FIGURE 10: Cross-polarized micrographs of samples illustrating the effect of TCB concentration and deposition speed with a unidirectional deposition method. This figure was reproduced with permission from [49]. Copyright 2014, Royal Society of Chemistry.

achieve highly crystalline films. While the traditional deposition techniques for organic electronics may lead to some interesting results, deposition techniques such as electrospinning, which allows readily depositing polymeric nanofibers on virtually any substrate, could also be worth exploring.

3.3. Aligned Molecules, Fibers, and Dipole Moments Using Electrospinning. Electrospinning is polymer nanofiber fabrication process which has been developing for around half a century and is often disregarded when it comes to device fabrication. The nanofibers are drawn from a polymer solution placed in a needle or capillary and deposited on a metal collector through electric forces (Figure 11). Electrospinning can generate continuous multilayer fiber mats or single layers depending on the processing conditions. For vertical device architecture electronics, smooth and continuous active layers are necessary which cannot be readily obtained by electrospinning. Nanofibers composed of polyethylene oxide (PEO) and poly(9,9-dioctylfluorenealt-benzothiadiazole) (F8BT) could be integrated in vertical architecture PLEDs by annealing the fibers after deposition which led to the extraction of PEO from the fibers generating a continuous isolating layer filling the gaps between the nanofibers [50]. Even though the PLEDs exhibit electroluminescence from the F8BT nanofibers, this can only be achieved for particular polymer/polymer combination and, therefore, cannot be considered as a general approach to deposit polymer electronics active layers using electrospinning.

Interestingly, nanofibers prepared using F8BT combined with PS show a high polarization ratio along the fiber axis $[50,51]$. The fibers could be used as down-conversion layers in hybrid inorganic-organic light-emitting devices, for instance, with a UV light-emitting device (inorganic) combined with polarized color generating electrospun nanofibers (organic) [52]. To fabricate polarized light sources using this strategy, fibers with extremely high dichroic ratios and that are deposited parallel to another should be produced. A large number of electrospinning set-ups have been proposed to obtain parallel deposited nanofiber mats. Some of these set-ups are represented in Figure 11. A simple strategy, which may be adaptable to roll-to-roll process, is to use a rotating drum collector on which the fiber undergoes a mechanical orientation resulting from the rotation of the drum. Alternatively, a number of collector designs have been proposed to readily deposit aligned nanofibers on fork-like collectors or parallel metal collector plates [53,54]. During 

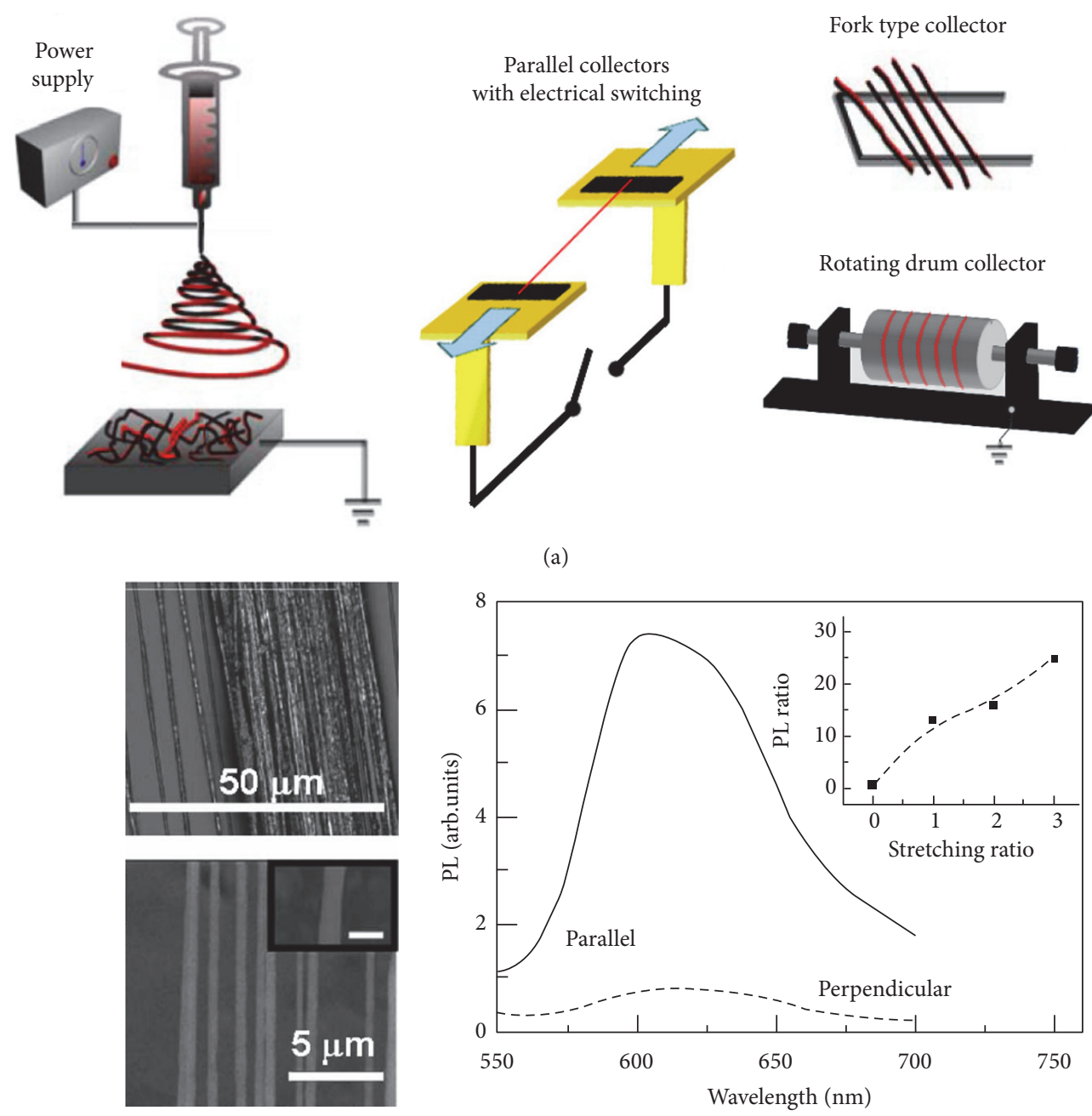

(b)

Figure 11: (a) Schematic representations of the traditional spin-coating set-up and alternatives to deposit oriented fibers. (b) Micrographs of aligned fibers and polarized photoluminescence obtained using the parallel collector set-up. This figure was adapted from [22, 53]. Copyright 2010, Royal Society of Chemistry. Copyright 2008, AIP Publishing LLC.

the process, the fibers undergo mechanical elongation along the fiber axis which results in conformational elongation of the polymer chains [55]. To obtain highly polarized fibers, an innovative set-up was developed by Ishii et al. in which electrical switching between two parallel collector plates allows for the fabrication of parallel free-standing nanofiber mats $[22,54]$. After deposition of the free-standing fibers, the distance between the parallel collector plates on which the fibers are anchored is increased in order to further mechanically elongate the polymer chains and orient them along the fiber axis. The micrographs and polarized photoluminescence spectra from the resulting fibers are presented in Figure 11. The photoluminescence polarization ratio (parallel to perpendicular to the fiber axis) of stretched nanofibers reaches values up to 25 .

Polarization of nanofibers is a valuable strategy for the generation of polarized light-emitting sources but can also be used in for other application. As mentioned in the previous part of this review, polarization of light can become a tool to control the amount of FRET from donor molecules with larger HOMO-LUMO bandgaps to lower energy acceptor molecules. Two studies on electrospun fibers containing dyeloaded zeolite $\mathrm{L}$ crystals practically demonstrate the potential of molecular orientation to control the emission properties of conjugated polymer based thin films and fibers [51, 56]. In these works, two types of nanofibers are used, namely, F8BT-based fibers which exhibit polarized emission along their fiber axis and poly(9,9-di-n-dodecylfluorenyl-2,7-diyl) (PFO) nanofibers with isotropic emission properties. The polarized F8BT fibers are combined with zeolites loaded with dyes that are also aligned along the fiber axis (Figure 12). This system generates a very efficient two-step FRET from F8BT to the red-emitting dye inside the zeolite crystals through an orange-emitting linking molecule (aligned at the entrance of the zeolite pores). Consequently, F8BT can be used to efficiently excite the dye within the zeolite crystal and the 


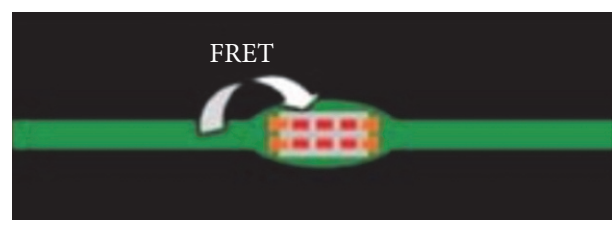

(a)

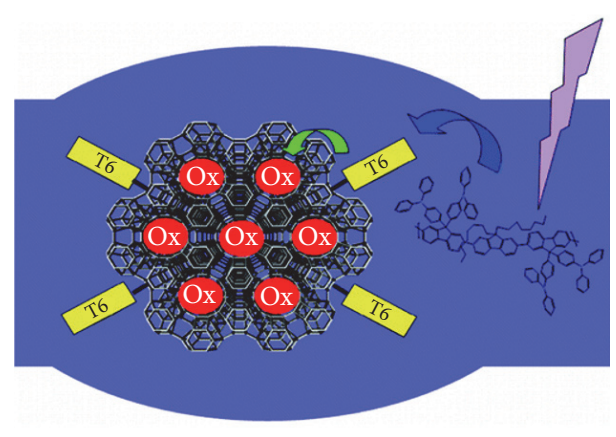

(c)

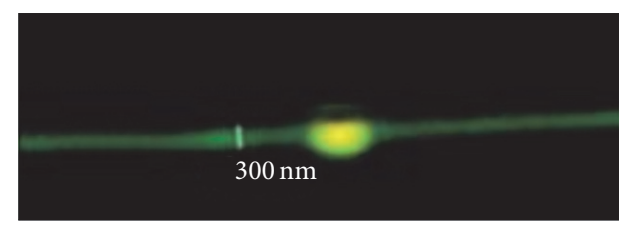

(b)

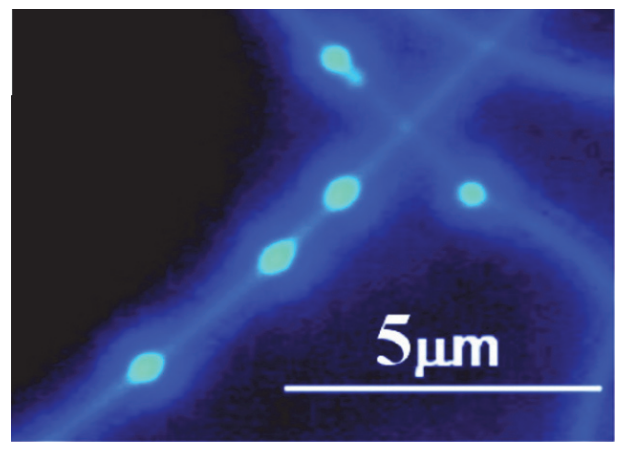

(d)

FIGURE 12: Schematic representations ( $\mathrm{a}$ and $\mathrm{c}$ ) and confocal micrographs (b and d) of polarized efficient (a and b) and controlled (c and d) FRET in zeolite L/conjugated polymer hybrid nanofibers. This figure was adapted from [51, 56]. Copyright 2009, John Wiley and Sons. Copyright 2010, American Chemical Society.

emission from the zeolite position in the confocal micrograph only exhibits photoluminescence from the two dyes included in the zeolite crystals (Figure 12).

On the other hand, PFO fibers were combined with randomly oriented dye-loaded nanozeolites. Both the conjugated polymer and the dyes included in the zeolite crystals did not display any polarized emission which led to partial FRET and, therefore, controlled emission from each molecule (blue, green, and red-emitting). This resulted in the possibility of fabricating white-light-emitting nanofibers with high photoluminescence quantum efficiencies, in which the color can be further tuned by simply adjusting the concentration of zeolite crystals in the fiber.

Note that, depending on the electrospinning conditions and, in particular, the nonconjugated polymer matrix used to assist the process of fiber formation, polarization along and across the fiber axis can be observed. By using coaxial electrospinning with an inner solution of $\mathrm{P} 3 \mathrm{HT}$ and an outer solution of poly (methyl methacrylate) (PMMA), Chen et al. demonstrated that molecular orientation of $\mathrm{P} 3 \mathrm{HT}$ within the fiber can be controlled to generate $\pi-\pi$ stacking of the conjugated polymer along the fiber axis [57]. The resulting fibers not only display highly polarized emission perpendicular to the fiber axis but also were used as active materials in PFETs. The highly oriented $(\pi-\pi$ stacking along the fiber axis) fibers exhibit field-effect mobilities which were 3 orders of magnitude higher than those obtained for nonoriented fibers using the same materials.

In summary, under the right conditions, electrospinning can naturally induce conjugated polymer orientation either along or parallel to the fiber axis which results in highly polarized emission properties from the conjugated polymer and/or enhanced field-effect mobilities. A particular attention should be given to the strategies to exploit those high performances in functional devices. Furthermore, the orientation can be enhanced by postdeposition mechanical processes. For instance, Pagliara et al. demonstrated that combining electrospinning with nanoimprinting can further enhance the polarized emission properties from nanofibers [58].

3.4. Molecular Orientation by Postdeposition Mechanical Processes. Applying postdeposition mechanical processes is not limited to electrospun fibers. Skrypnychuk et al. demonstrated that imprinting can be applied to spin-coated P3HT films generating $1 \mu \mathrm{m}$ high and $4 \mu \mathrm{m}$ wide arrays of pillar like structures [59]. Unlike smooth films which contain mainly edge-on crystallites, the formation of micropillars induces preferential orientation of the $\mathrm{P} 3 \mathrm{HT}$ chains with mainly chain-on crystallite orientation (Figure 13). The films were integrated into a simple vertical device (micropillars or smooth films sandwiched between two electrodes) to extract the hole mobilities in smooth and micropattern active layers. The mobility values for the smooth films were on the order of $10^{-4} \mathrm{~cm}^{2} \cdot \mathrm{V}^{-1} \cdot \mathrm{s}^{-1}$, which is consistent with other studies on P3HT vertical architecture hole-only devices. Upon microstructuration, the hole mobility increased more than 4 orders of magnitude to reach values of approximately $3 \mathrm{~cm} \cdot \mathrm{V}^{-1} \cdot \mathrm{s}^{-1}$. This large increase in mobility was ascribed to a large contribution from charge transport along the polymer backbone in the vertical architecture chain-on P3HT devices. Although molecular reorientation is commonly associated with confinement on the nanometer scale, here, microconfinement exhibited high molecular reorientation abilities. Similar effect could be observed when using nanoconfinement (nanoscale features) for both $\mathrm{P} 3 \mathrm{HT}$ and other polymers 


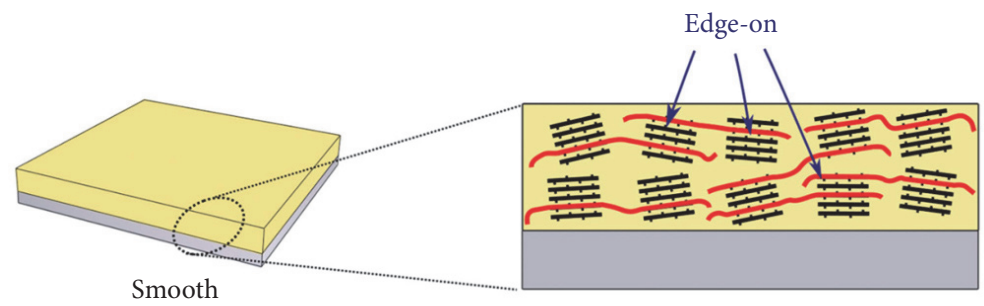

(a)

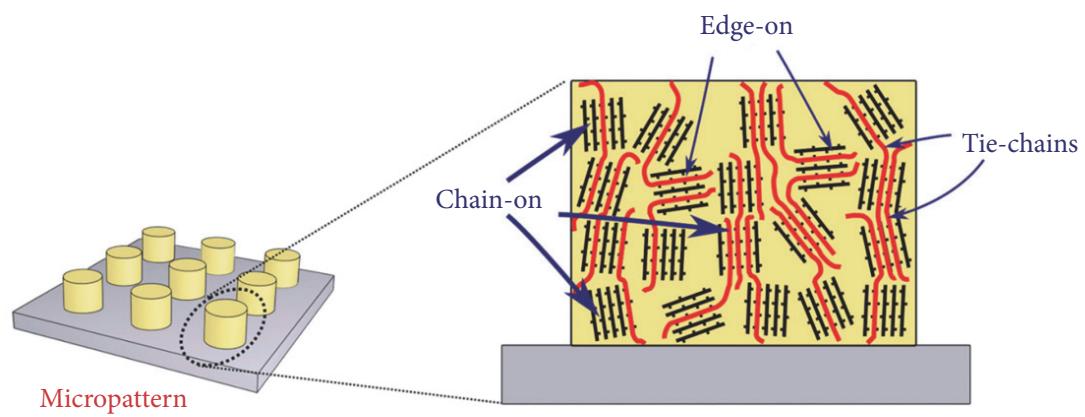

(b)

FIGURE 13: Schematic representations of smooth and micropatterned P3HT films and their crystallite orientation. This figure was reproduced with permission from [59]. Copyright 2016, John Wiley and Sons.

in which orientations can be controlled by nanodimensions and experimental conditions [23, 60-64].

In fact, depending on the shape of the structure used for nanoimprinting, unidirectional molecular orientation can also be achieved (Figure 14) [60]. Zheng et al. used triangular structures to induce such unidirectional orientation on F8BT and integrated the oriented thin films into PFET and PLED devices. The molecularly oriented films displayed anisotropy not only in optical properties but also in hole and electron field-effect mobilities. The anisotropy for mobilities along and perpendicular to the molecular orientation direction reached values over 10 and 5, respectively, for hole and electron mobilities. Furthermore, the PLEDs fabricated using nanoimprinted F8BT active layers exhibited strongly polarized electroluminescence along the polymer orientation direction (Figure 14).

Another efficient method to orient molecules using postdeposition mechanical processes is called rubbing. It is a well-known technique applied in the field of LCDs. 2D-XRD measurements revealed that rubbing P3HT layers generates molecular order whether the films are rubbed with velvet or nylon materials (Figure 15) [33, 65-68]. In particular, depending on the temperature at which the rubbing process is performed, the amount of face-on and edge-on crystallites can be controlled to induce the adequate crystallite orientation depending on the application [66]. For instance, the vertical hole mobility can be enhanced about twofold with the generation of face-on crystallites and mobility anisotropy can also be generated through rubbing [65-67]. Although most of the studies on rubbing focus on $\mathrm{P} 3 \mathrm{HT}$, this process is not limited to thiophene-based polymers. In fact, the original technique applied in LCD fabrication consists in rubbing PMMA or poly(imide) (PI) thin films which are then used as templates to induce molecular orientation in the subsequently deposited layer. This process was adapted to deposit highly oriented F8BT chains on rubbed PI covered ITO substrates and integrate them into PLEDs (Figure 16) [69]. Rubbing the PI layer 7 times resulted in dichroic ratios in the F8BT layer over 20 and the generation of highly polarized PLEDs.

Rubbing can also be performed directly on the active layer materials in PLEDs or PSCs $[17,18,68]$. By doing so, innovative devices and concepts can be produced, once again, emphasizing the large potential of conjugated polymer materials when it comes to process and device integration. Sequential deposition and rubbing of two active conjugated polymer layers rubbed orthogonally were performed using poly(phenylene vinylene) (PPV) and poly(thiophene) derivative active molecules [17]. After deposition of PPV precursors, these were rubbed to generate unidirectional orientation in the bottom part of the active layer. Annealing the PPV precursors, making them unsoluble in common organic solvents, allowed for the deposition of a second active layer using spin-coating. The second layer (top part of the active layer), in this case composed of a red-emitting poly(thiophene) derivative, was then rubbed in a direction perpendicular to that of the first layer to generate bipolarized active layers which are integrated into a PLED architecture. The resulting device exhibited bipolarized electroluminescence with the possibility to easily switch from a red emission to a greenyellowish one by simply rotating a polarizer placed on top of the device (Figure 17). On the other hand, by applying rubbing to the active layer of PSCs, the strongly oriented 


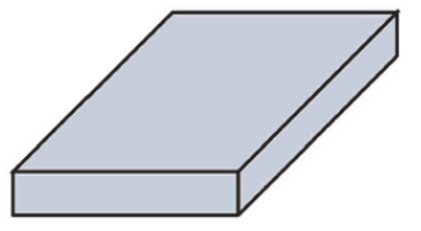

I $\downarrow \begin{gathered}\text { Spin-coat } \\ \text { F8BT }\end{gathered}$

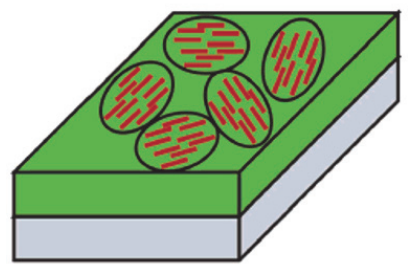

II $\downarrow$ Nanoimprint

- Substrate

- Conjugated backbone
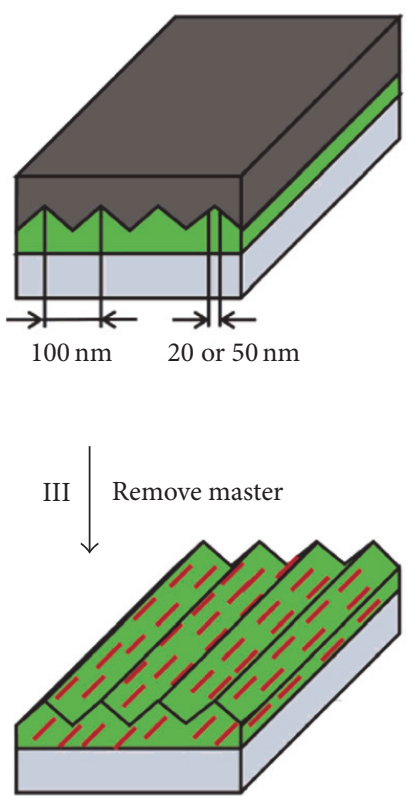

F8BT

Si master
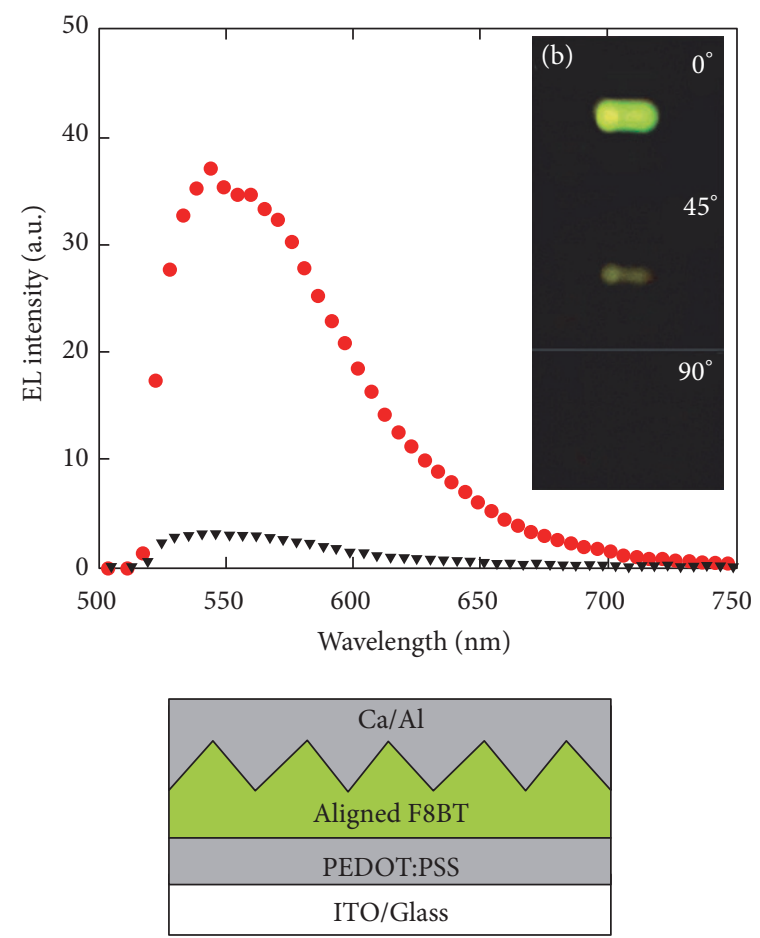

FIGURE 14: Schematic representation of the formation of aligned F8BT thin films and PLEDS along with their polarized electroluminescence properties. This figure was adapted with permission from [60]. Copyright 2007, American Chemical Society.
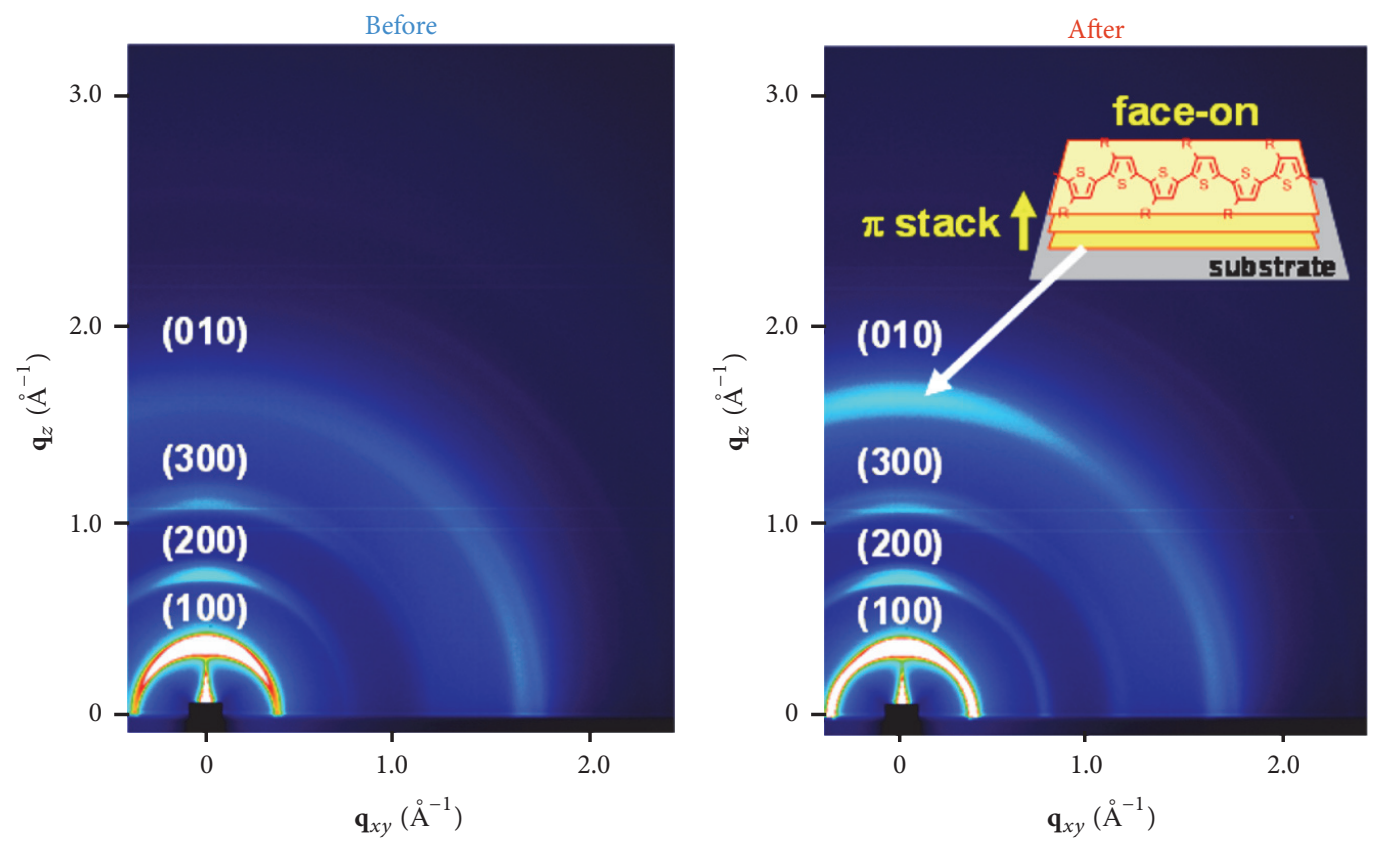

FIGURE 15: 2D-XRD images of P3HT films before and after rubbing. Figure reproduced with permission from [33]. Copyright 2015, American Chemical Society.

P3HT molecules only absorb the light polarized along one direction and leave the remaining light to pass through the semitransparent devices. The working principle of LCDs is based on selective absorption of polarized light to orient the liquid crystal molecules sandwiched between two polarizers.
Zhu et al. exploited the peculiar properties of rubbed PSCs and LCDs to combine the two devices and fabricate a new generation of LCD in which the light which is commonly lost through absorption by the polarizers can be used for energy production from the integrated PSCs (Figure 17) [18]. 


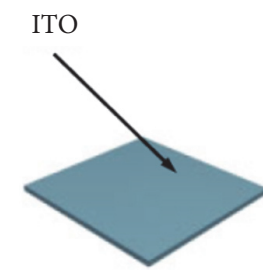

(a)

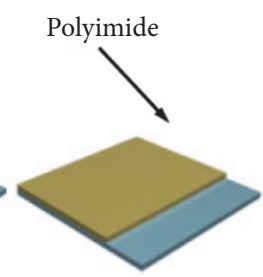

(b)

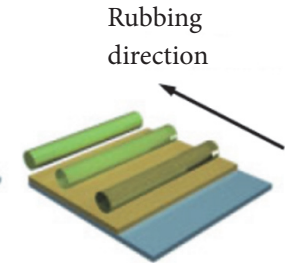

(c)

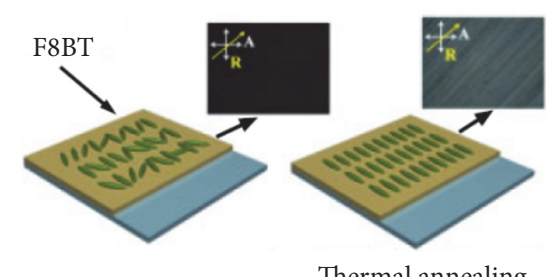

(d)
Thermal annealing

(e)

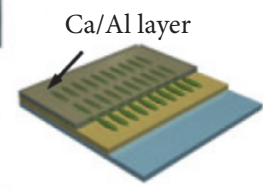

(f)

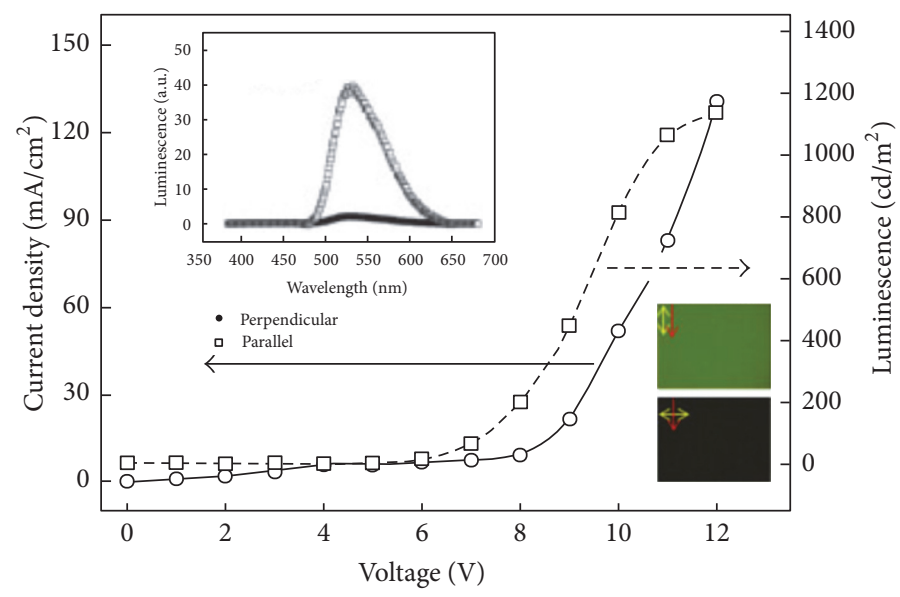

FIgURE 16: Schematic representation of the PI rubbing process inducing F8BT alignment in PLEDs active layers and the resulting device performances and polarized electroluminescence from these films. Figure adapted with permission from [65]. Copyright 2012, The Japan Society of Applied Physics.
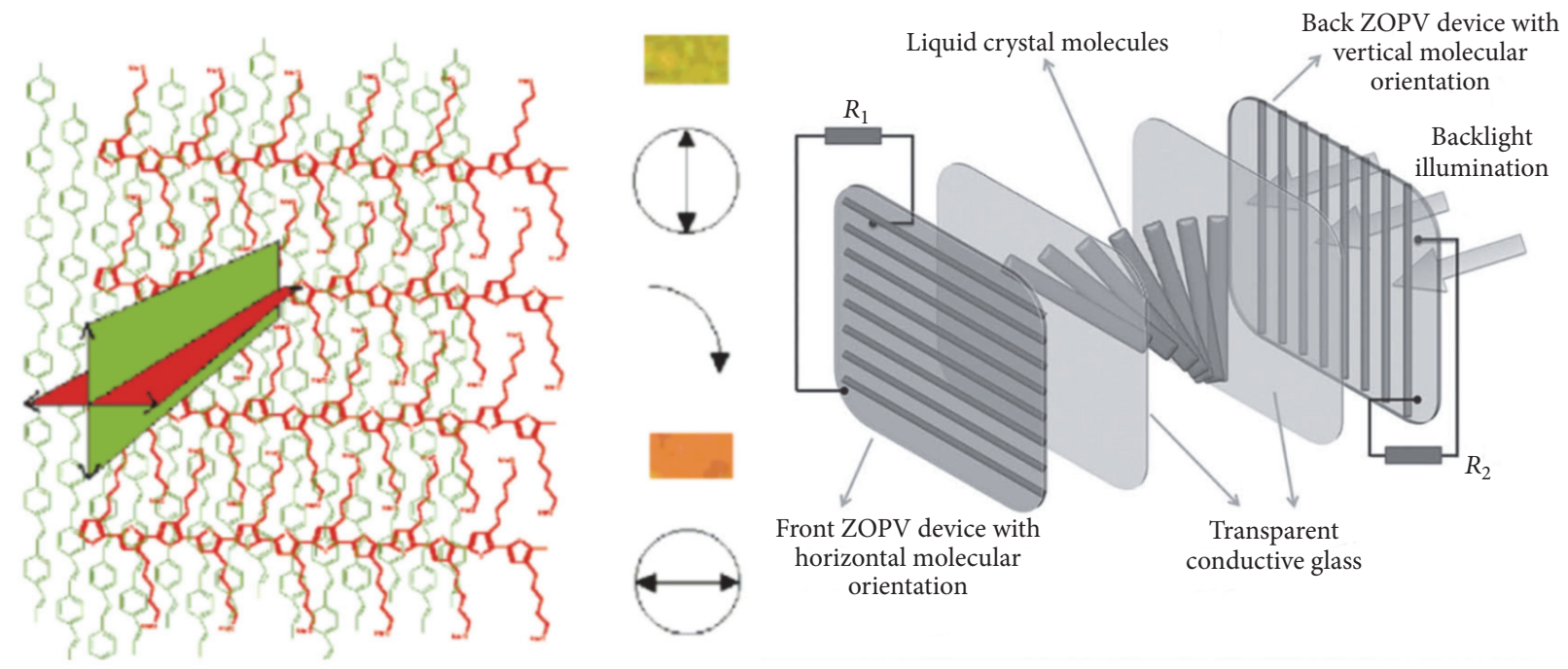

FIGURE 17: Innovative device designs based on the ingenuous use of perpendicularly polarized light. This figure was adapted with permission from [17, 18]. Copyrights 2001 and 2011, John Wiley and Sons.

Although postdeposition process results in additional steps for device production, the high performances of the fabricated devices and the possibility to produce original concept devices clearly demonstrate that they can be considered as one of the most efficient and versatile processes for molecular orientation for optoelectronic applications.

\section{Conclusions}

In summary, conjugated polymers have the potential to revolutionize the future of optoelectronic technologies. Due to their peculiar mechanical properties, a large number of processes can be applied to generate molecular orientation, which enhances not only the optical properties in thin films and nanofibers but also the charge transport properties (vertical charge transport or field-effect lateral mobility). Among the numerous processes available for molecular orientation of conjugated polymers, we have observed that particularly interesting results can be obtained with surface/interface engineering, epitaxial growth, mechanical processes (rubbing, elongation in electrospun nanofibers), and nanoconfinement. In fact, PFETs fabricated with uniaxially oriented conjugated polymer chains display anisotropy in mobility over 30. Similarly, chain orientation induces both anisotropy in absorption, photoluminescence, and electroluminescence in light-emitting thin films and nanofibers 
with ratios often exceeding a value of 20. Furthermore, the adequate crystallite orientation in PFETs and PSCs resulted in the fabrication of devices with record efficiencies (mobilities up to $43 \mathrm{~cm}^{2} \cdot \mathrm{V}^{-1} \cdot \mathrm{s}^{-1}$ and PCE over $10 \%$, resp.). Interactions between polarized light and oriented conjugated polymer molecules can also be exploited to develop new device concepts such as integrated PSCs LCDs or color-switchable PLEDs. These results not only confirm the potential of conjugated polymers as materials for future technology fabrication but also demonstrate that a particular attention should be given to molecular orientation and crystallinity of the polymer chains to produce high performances and innovative devices.

\section{Conflicts of Interest}

The authors declare that they have no conflicts of interest regarding the publication of this paper.

\section{References}

[1] P.-O. Morin, T. Bura, and M. Leclerc, "Realizing the full potential of conjugated polymers: innovation in polymer synthesis," Materials Horizons, vol. 3, no. 1, pp. 11-20, 2016.

[2] A. Facchetti, " $\pi$-Conjugated polymers for organic electronics and photovoltaic cell applications," Chemistry of Materials, vol. 23, no. 3, pp. 733-758, 2011.

[3] R. R. Søndergaard, M. Hösel, and F. C. Krebs, "Roll-to-Roll fabrication of large area functional organic materials," Journal of Polymer Science, Part B: Polymer Physics, vol. 51, no. 1, pp. 1634, 2013.

[4] M. Brinkmann, L. Hartmann, L. Biniek, K. Tremel, and N. Kayunkid, "Orienting semi-conducting $\pi$-conjugated polymers," Macromolecular Rapid Communications, vol. 35, no. 1, pp. 9-26, 2014.

[5] R. J. Kline and M. D. McGehee, "Morphology and charge transport in conjugated polymers," Polymer Reviews, vol. 46, no. 1, pp. 27-45, 2006.

[6] I. Botiz and N. Stingelin, "Influence of molecular conformations and microstructure on the optoelectronic properties of conjugated polymers," Materials, vol. 7, no. 3, pp. 2273-2300, 2014.

[7] I. Botiz, S. Astilean, and N. Stingelin, "Altering the emission properties of conjugated polymers," Polymer International, vol. 65, no. 2, pp. 157-163, 2016.

[8] A. D. Printz and D. J. Lipomi, "Competition between deformability and charge transport in semiconducting polymers for flexible and stretchable electronics," Applied Physics Reviews, vol. 3, no. 2, Article ID 021302, 2016.

[9] R. Noriega, J. Rivnay, K. Vandewal et al., "A general relationship between disorder, aggregation and charge transport in conjugated polymers," Nature Materials, vol. 12, no. 11, pp. 1038-1044, 2013.

[10] S. A. Mollinger, B. A. Krajina, R. Noriega, A. Salleo, and A. J. Spakowitz, "Percolation, tie-molecules, and the microstructural determinants of charge transport in semicrystalline conjugated polymers," ACS Macro Letters, vol. 4, no. 7, pp. 708-712, 2015.

[11] V. Coropceanu, J. Cornil, D. A. da Silva Filho, Y. Olivier, R. Silbey, and J.-L. Brédas, "Charge transport in organic semiconductors,” Chemical Reviews, vol. 107, no. 4, pp. 926-952, 2007.
[12] D. Alberga, A. Perrier, I. Ciofini, G. F. Mangiatordi, G. Lattanzi, and C. Adamo, "Morphological and charge transport properties of amorphous and crystalline P3HT and PBTTT: insights from theory," Physical Chemistry Chemical Physics, vol. 17, no. 28, pp. 18742-18750, 2015.

[13] E. Matioli, S. Brinkley, K. M. Kelchner et al., "High-brightness polarized light-emitting diodes," Light: Science and Applications, vol. 1, article e22, 2012.

[14] H. K. Park, S. W. Yoon, Y. J. Eo et al., "green In GaN nanorod LEDs: scalable polarized surface emitting LEDs using electricfield assisted assembly," Scientific Reports, vol. 6, Article ID 28312, 2016.

[15] B. Park, Y. H. Huh, and H. G. Jeon, "Electroluminescence from organic light-emitting devices using photon recycling," Optics Express, vol. 18, no. 19, pp. 19824-19830, 2010.

[16] R. Ding, J. Feng, W. Zhou et al., "Intrinsic polarization and tunable color of electroluminescence from organic single crystalbased light-emitting devices," Scientific Reports, vol. 5, Article ID 12445, 2015.

[17] A. Bolognesi, C. Botta, D. Facchinetti et al., "Polarized electroluminescence in double-layer light-emitting diodes with perpendicularly oriented polymers," Advanced Materials, vol. 13, no. 14, pp. 1072-1075, 2001.

[18] R. Zhu, A. Kumar, and Y. Yang, "Polarizing organic photovoltaics," Advanced Materials, vol. 23, no. 36, pp. 4193-4198, 2011.

[19] M. Brinkmann and J.-C. Wittmann, "Orientation of regioregular poly(3-hexylthiophene) by directional solidification: a simple method to reveal the semicrystalline structure of a conjugated polymer," Advanced Materials, vol. 18, no. 7, pp. 860863, 2006.

[20] D. Natali, M. Sampietro, L. Franco, A. Bolognesi, and C. Botta, "Mobility anisotropy in Langmuir-Blodgett deposited poly(3-methoxypentyl-tiophene)-based thin film transistors," Thin Solid Films, vol. 472, no. 1-2, pp. 238-241, 2005.

[21] N.-K. Kim, S.-Y. Jang, G. Pace et al., "High-performance organic field-effect transistors with directionally aligned conjugated polymer film deposited from pre-aggregated solution," Chemistry of Materials, vol. 27, no. 24, pp. 8345-8353, 2015.

[22] M. Campoy-Quiles, Y. Ishii, H. Sakai, and H. Murata, "Highly polarized luminescence from aligned conjugated polymer electrospun nanofibers," Applied Physics Letters, vol. 92, no. 21, Article ID 213305, 2008.

[23] Z. Hu and A. M. Jonas, "Control of crystal orientation in soft nanostructures by nanoimprint lithography," Soft Matter, vol. 6, no. 1, pp. 21-28, 2009.

[24] W.-S. Hu, S.-Z. Weng, Y.-T. Tao, H.-J. Liu, and H.-Y. Lee, "Oriented growth of rubrene thin films on aligned pentacene buffer layer and its anisotropic thin-film transistor characteristics," Organic Electronics, vol. 9, no. 3, pp. 385-395, 2008.

[25] V. Vohra, K. Kawashima, T. Kakara et al., "Efficient inverted polymer solar cells employing favourable molecular orientation," Nature Photonics, vol. 9, no. 6, pp. 403-408, 2015.

[26] I. Osaka, M. Shimawaki, H. Mori et al., "Synthesis, characterization, and transistor and solar cell applications of a naphthobisthiadiazole-based semiconducting polymer," Journal of the American Chemical Society, vol. 134, no. 7, pp. 34983507, 2012.

[27] T. Matsushima and H. Murata, "Enhancement of hole injection and electroluminescence characteristics by a rubbing-induced lying orientation of alpha-sexithiophene," Journal of Applied Physics, vol. 112, no. 2, Article ID 024503, 2012. 
[28] D. Yokoyama, "Molecular orientation in small-molecule organic light-emitting diodes," Journal of Materials Chemistry, vol. 21, no. 48, pp. 19187-19202, 2011.

[29] R. Ding, J. Feng, X.-L. Zhang et al., "Fabrication and characterization of organic single crystal-based light-emitting devices with improved contact between the metallic electrodes and crystal," Advanced Functional Materials, vol. 24, no. 45, pp. 7085-7092, 2014.

[30] M. S. Vezie, S. Few, I. Meager et al., "Exploring the origin of high optical absorption in conjugated polymers," Nature Materials, vol. 15, no. 7, pp. 746-753, 2016.

[31] J. Xu and Z. Guo, "Biomimetic photonic materials with tunable structural colors," Journal of Colloid and Interface Science, vol. 406, pp. 1-17, 2013.

[32] V. Vohra, K. Higashimine, T. Murakami, and H. Murata, "Addition of regiorandom poly(3-hexylthiophene) to solution processed poly(3-hexylthiophene):[6,6]-phenyl-C61-butyric acid methyl ester graded bilayers to tune the vertical concentration gradient," Applied Physics Letters, vol. 101, no. 17, Article ID 173301, 2012.

[33] D. Kajiya, S. Ozawa, T. Koganezawa, and K.-I. Saitow, "Enhancement of out-of-plane mobility in P3HT film by rubbing: aggregation and planarity enhanced with low regioregularity," Journal of Physical Chemistry C, vol. 119, no. 15, pp. 7987-7995, 2015.

[34] G. Bongiovanni, C. Botta, G. Di Silvestro, M. A. Loi, A. Mura, and R. Tubino, "Energy transfer in nanostructured oligothiophene inclusion compounds," Chemical Physics Letters, vol. 345, no. 5-6, pp. 386-394, 2001.

[35] C.-Y. Lu, M. Jiao, W.-K. Lee et al., "Achieving above 60\% external quantum efficiency in organic light-emitting devices using ITO-free low-index transparent electrode and emitters with preferential horizontal emitting dipoles," Advanced Functional Materials, vol. 26, no. 19, pp. 3250-3258, 2007.

[36] D. H. Kim, Y. D. Park, Y. Jang et al., "Enhancement of fieldeffect mobility due to surface-mediated molecular ordering in regioregular polythiophene thin film transistors," Advanced Functional Materials, vol. 15, no. 1, pp. 77-82, 2005.

[37] V. Vohra, M. Campoy-Quiles, M. Garriga, and H. Murata, "Organic solar cells based on nanoporous P3HT obtained from self-assembled P3HT:PS templates," Journal of Materials Chemistry, vol. 22, no. 37, pp. 20017-20025, 2012.

[38] Y. Lei, P. Deng, J. Li et al., "Solution-processed donoracceptor polymer nanowire network semiconductors for highperformance field-effect transistors," Scientific Reports, vol. 6, Article ID 24476, 2016.

[39] L. Qiu, X. Wang, W. H. Lee et al., "Organic thin-film transistors based on blends of poly(3-hexylthiophene) and polystyrene with a solubility-induced low percolation threshold," Chemistry of Materials, vol. 21, no. 19, pp. 4380-4386, 2009.

[40] V. Vohra, B. Dörling, K. Higashimine, and H. Murata, "Investigating the effect of solvent boiling temperature on the active layer morphology of diffusive bilayer solar cells," Applied Physics Express, vol. 9, no. 1, Article ID 012301, 2016.

[41] C. Müller, M. Aghamohammadi, S. Himmelberger et al., "Onestep macroscopic alignment of conjugated polymer systems by epitaxial crystallization during spin-coating," Advanced Functional Materials, vol. 23, no. 19, pp. 2368-2377, 2013.

[42] J. Y. Kim, D. S. Yang, J. Shin et al., "High-performing thin-film transistors in large spherulites of conjugated polymer formed by epitaxial growth on removable organic crystalline templates,"
ACS Applied Materials and Interfaces, vol. 7, no. 24, pp. 1343113439, 2015.

[43] A. Bolognesi, G. Bajo, J. Paloheimo, T. Ostergard, and H. Stubb, "Polarized electroluminescence from an oriented poly(3alkylthiophene) Langmuir-Blodgett structure," Advanced Materials, vol. 9, no. 2, pp. 121-124, 1997.

[44] V. Cimrová, M. Remmers, D. Neher, and G. Wegner, "Polarized light emission from LEDs prepared by the langmuir-blodgett technique," Advanced Materials, vol. 8, no. 2, pp. 146-149, 1996.

[45] Y. Yuan, G. Giri, A. L. Ayzner et al., "Ultra-high mobility transparent organic thin film transistors grown by an off-centre spin-coating method," Nature Communications, vol. 5, article 3005,2014

[46] J. Huang, J. H. Carpenter, C.-Z. Li, J.-S. Yu, H. Ade, and A. K.-Y. Jen, "Highly efficient organic solar cells with improved vertical donor-acceptor compositional gradient via an inverted off-center spinning method," Advanced Materials, vol. 28, no. 5, pp. 967-974, 2016.

[47] L. Shaw, P. Hayoz, Y. Diao et al., "Direct uniaxial alignment of a donor-acceptor semiconducting polymer using single-step solution shearing," ACS Applied Materials and Interfaces, vol. 8, no. 14, pp. 9285-9296, 2016.

[48] S. N. Patel, G. M. Su, C. Luo et al., "NEXAFS spectroscopy reveals the molecular orientation in blade-coated pyridal[2,1,3]thiadiazole-containing conjugated polymer thin films," Macromolecules, vol. 48, no. 18, pp. 6606-6616, 2015.

[49] B. Dörling, V. Vohra, T. T. Dao, M. Garriga, H. Murata, and M. Campoy-Quiles, "Uniaxial macroscopic alignment of conjugated polymer systems by directional crystallization during blade coating," Journal of Materials Chemistry C, vol. 2, no. 17, pp. 3303-3310, 2014.

[50] V. Vohra, U. Giovanella, R. Tubino, H. Murata, and C. Botta, "Electroluminescence from conjugated polymer electrospun nanofibers in solution processable organic light-emitting diodes," ACS Nano, vol. 5, no. 7, pp. 5572-5578, 2011.

[51] V. Vohra, A. Devaux, L.-Q. Dieu et al., "Energy transfer in fluorescent nanofibers embedding dye-loaded zeolite l crystals," Advanced Materials, vol. 21, no. 10-11, pp. 1146-1150, 2009.

[52] F. Galeotti, W. Mróz, M. Catellani, B. Kutrzeba-Kotowska, and E. Kozma, "Tailorable perylene-loaded fluorescent nanostructures: a multifaceted approach enabling their application in white hybrid LEDs," Journal of Materials Chemistry C, vol. 4, no. 23, pp. 5407-5415, 2016.

[53] K. Yin, L. Zhang, C. Lai et al., "Photoluminescence anisotropy of uni-axially aligned electrospun conjugated polymer nanofibers of MEH-PPV and P3HT,' Journal of Materials Chemistry, vol. 21, no. 2, pp. 444-448, 2011.

[54] Y. Ishii, H. Sakai, and H. Murata, "A new electrospinning method to control the number and a diameter of uniaxially aligned polymer fibers," Materials Letters, vol. 62, no. 19, pp. 3370-3372, 2008.

[55] A. Camposeo, I. Greenfeld, F. Tantussi et al., "Conformational evolution of elongated polymer solutions tailors the polarization of light-emission from organic nanofibers," Macromolecules, vol. 47, no. 14, pp. 4704-4710, 2014.

[56] V. Vohra, G. Calzaferri, S. Destri, M. Pasini, W. Porzio, and C. Botta, "Toward white light emission through efficient two-step energy transfer in hybrid nanofibers," ACS Nano, vol. 4, no. 3, pp. 1409-1416, 2010.

[57] J.-Y. Chen, C.-C. Kuo, C.-S. Lai, W.-C. Chen, and H.-L. Chen, "Manipulation on the morphology and electrical properties of 
aligned electrospun nanofibers of poly(3-hexylthiophene) for field-effect transistor applications," Macromolecules, vol. 44, no. 8, pp. 2883-2892, 2011.

[58] S. Pagliara, A. Camposeo, E. Mele, L. Persano, R. Cingolani, and D. Pisignano, "Enhancement of light polarization from electrospun polymer fibers by room temperature nanoimprint lithography," Nanotechnology, vol. 21, no. 21, Article ID 215304, 2010.

[59] V. Skrypnychuk, G.-J. A. H. Wetzelaer, P. I. Gordiichuk et al., "Ultrahigh mobility in an organic semiconductor by vertical chain alignment," Advanced Materials, vol. 28, no. 12, pp. 23592366, 2016.

[60] Z. Zheng, K.-H. Yim, M. S. M. Saifullah et al., "Uniaxial alignment of liquid-crystalline conjugated polymers by nanoconfinement," Nano Letters, vol. 7, no. 4, pp. 987-992, 2007.

[61] M. Aryal, K. Trivedi, and W. Hu, "Nano-confinement induced chain alignment in ordered $\mathrm{P} 3 \mathrm{HT}$ nanostructures defined by nanoimprint lithography," ACS Nano, vol. 3, no. 10, pp. 30853090, 2009.

[62] H. Hlaing, X. Lu, T. Hofmann, K. G. Yager, C. T. Black, and B. M. Ocko, "Nanoimprint-induced molecular orientation in semiconducting polymer nanostructures," ACS Nano, vol. 5, no. 9, pp. 7532-7538, 2011.

[63] S. Kushi, R. Tsukada, K. Noguchi, and T. Shimomura, "Crystallization of poly(3-hexylthiophene) nanofiber in a narrow groove," Polymers, vol. 8, no. 6, article 231, 2016.

[64] X. Lu, H. Hlaing, C.-Y. Nam, K. G. Yager, C. T. Black, and B. M. Ocko, "Molecular orientation and performance of nanoimprinted polymer-based blend thin film solar cells," Chemistry of Materials, vol. 27, no. 1, pp. 60-66, 2015.

[65] L. Hartmann, K. Tremel, S. Uttiya et al., "2D versus 3D crystalline order in thin films of regioregular poly(3-hexylthiophene) oriented by mechanical rubbing and epitaxy," Advanced Functional Materials, vol. 21, no. 21, pp. 4047-4057, 2011.

[66] A. Hamidi-Sakr, L. Biniek, S. Fall, and M. Brinkmann, "Precise control of lamellar thickness in highly oriented regioregular poly(3-hexylthiophene) thin films prepared by hightemperature rubbing: correlations with optical properties and charge transport," Advanced Functional Materials, vol. 26, no. 3, pp. 408-420, 2016.

[67] L. Biniek, S. Pouget, D. Djurado et al., "High-temperature rubbing: a versatile method to align $\pi$-conjugated polymers without alignment substrate," Macromolecules, vol. 47, no. 12, pp. 3871-3879, 2014.

[68] V. Vohra, G. Arrighetti, L. Barba, K. Higashimine, W. Porzio, and H. Murata, "Enhanced vertical concentration gradient in rubbed P3HT:PCBM graded bilayer solar cells," Journal of Physical Chemistry Letters, vol. 3, no. 13, pp. 1820-1823, 2012.

[69] S. I. Jo, Y. Kim, J.-H. Baek, C.-J. Yu, and J.-H. Kim, "Highly polarized emission of the liquid crystalline conjugated polymer by controlling the surface anchoring energy," Japanese Journal of Applied Physics, vol. 53, no. 3, Article ID 03CD04, 2014.

[70] M. Brinkmann, "Epitaxy of conjugated semi-conducting polymers: a simple method to prepare oriented and nanostructured organic and hybrid materials," L’Actualité chimique, vol. 326, pp. 31-34, 2009. 

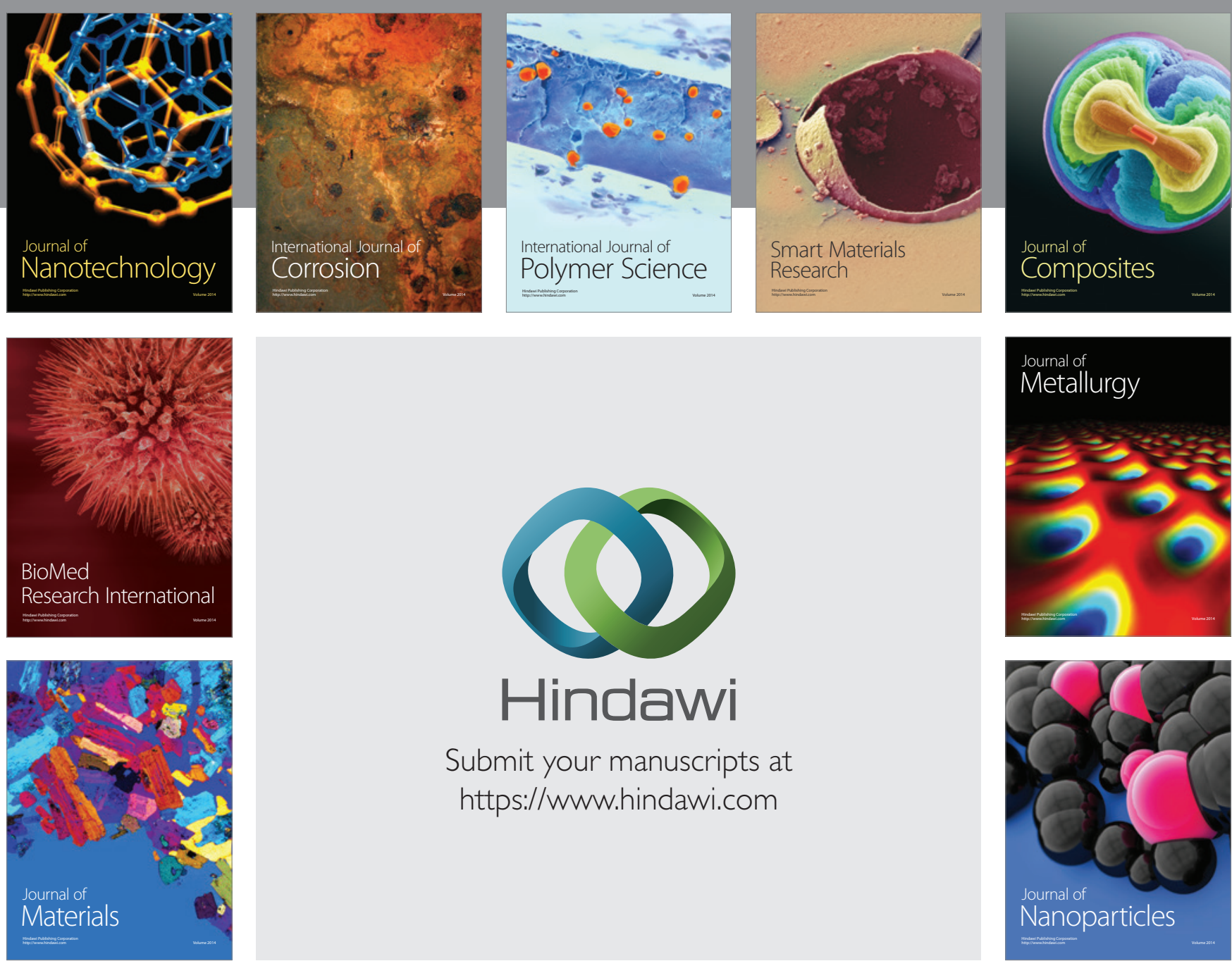

\section{Hindawi}

Submit your manuscripts at

https://www.hindawi.com

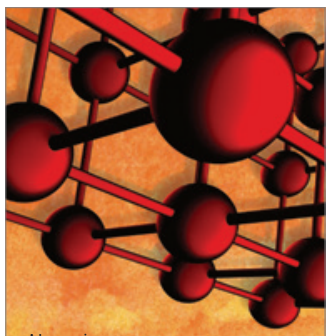

Materials Science and Engineering
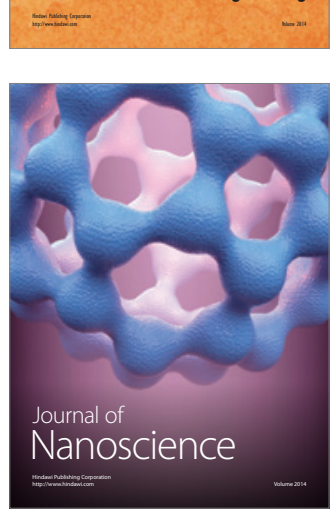
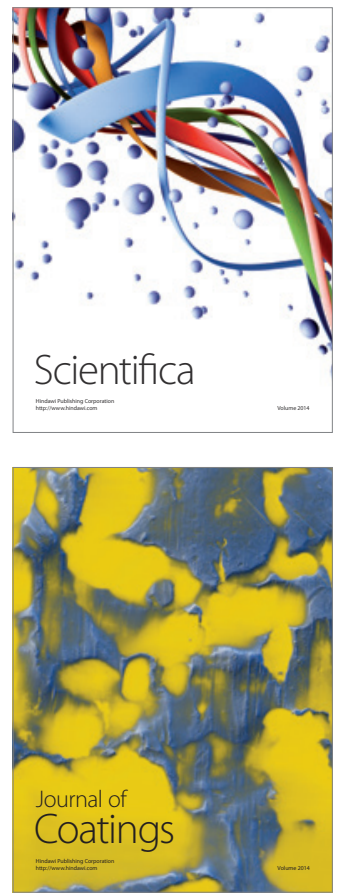
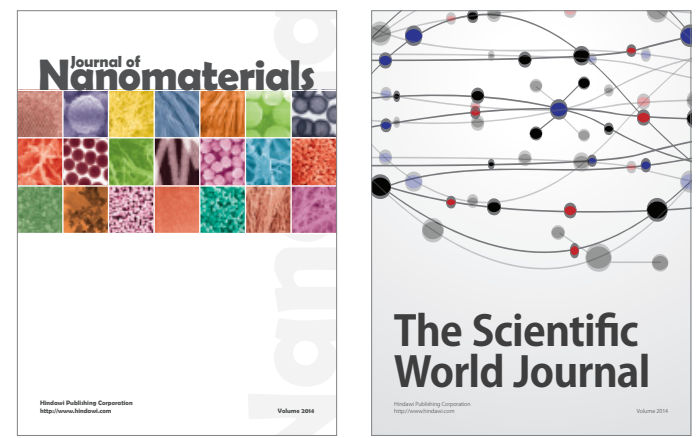

The Scientific World Journal
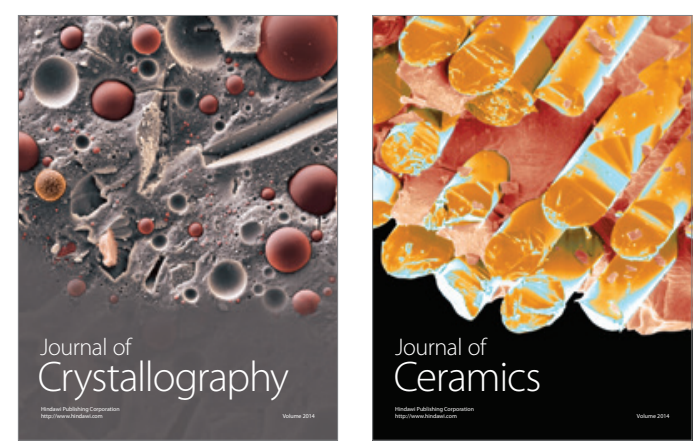
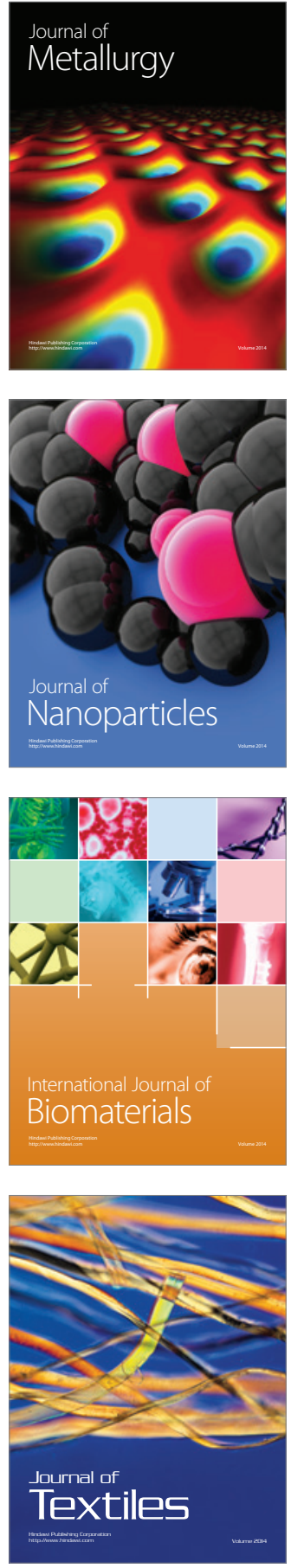